\title{
Listeria monocytogenes TcyKLMN cystine/cysteine transporter facilitates glutathione synthesis and virulence gene expression
}

Moran Brenner ${ }^{1}$, Sivan Friedman ${ }^{1}$, Adi Haber ${ }^{2}$, Ilya Borovok ${ }^{1}$, Nadejda Sigal ${ }^{1}$, Oded 5

Lewinson $^{2}$, and Anat A. Herskovits ${ }^{1 *} \quad 6$

${ }^{1}$ The Shmunis School of Biomedicine and Cancer Research, The George S. Wise Faculty of 8

Life Sciences, Tel Aviv University, Tel Aviv, Israel 9

${ }^{2}$ Department of Biochemistry, The Bruce and Ruth Rappaport Faculty of Medicine, The 10

Rappaport Institute for Biomedical research, Technion-Israel Institute of Technology, Haifa, 11

$\begin{array}{ll}\text { Israel } & 12\end{array}$

*To whom correspondence should be addressed: e-mail: anathe@tauex.tau.ac.il

Keywords: Listeria monocytogenes, glutathione biosynthesis, GSH, cystine/cysteine

The Shmunis School of Biomedicine and Cancer Research,

The George S. Wise Life Sciences Faculty

Tel Aviv University, Tel Aviv, Israel, 69978

Phone: 972-3-640-7502

Fax: 972-3-640-9407 


\begin{abstract}
Listeria monocytogenes $(\mathrm{Lm})$ is a saprophyte and a human intracellular pathogen. Up27 on invasion into mammalian cells, it senses multiple metabolic and environmental 28 signals that collectively trigger its transition to the pathogenic state. One of these sig29 nals is the tripeptide glutathione, which acts as an allosteric activator of $\mathrm{Lm}$ 's master 30 virulence regulator, PrfA. While glutathione synthesis by $L m$ was shown to be critical 31 for PrfA activation and virulence gene expression, it remains unclear how this tripep32 tide is synthesized under changing environments, especially in light of the observation 33 that $L m$ is auxotrophic to one of its precursors, cysteine. Here, we show that the ABC transporter TcyKLMN is a cystine/cysteine importer that supplies cysteine for glutathione synthesis, hence mediating the induction of the virulence genes. Further, we demonstrate that this transporter is negatively regulated by three metabolic regulators: CodY, CymR and CysK, which sense and respond to changing concentrations of branched chain amino acids (BCAA) and cysteine. The data indicate that under low 39 concentrations of BCAA, TcyKLMN is up-regulated, driving the production of glutathione by supplying cysteine, thereby facilitating PrfA activation. These findings provide molecular insight into the coupling of $\mathrm{Lm}$ metabolism and virulence, connecting BCAA sensing to cysteine uptake and glutathione biosynthesis, as a mechanism that controls virulence gene expression. This study exemplifies how bacterial pathogens sense their intracellular environment and exploit essential metabolites as effectors of virulence.

\title{
Importance
}

Bacterial pathogens sense the repertoire of metabolites in the mammalian niche and 49 use this information to shift into a pathogenic state to accomplish successful infection.

Glutathione is a virulence-activating signal that is synthesized by L. monocytogenes during infection of mammalian cells. In this study, we show that cysteine uptake via TcyKLMN drives glutathione synthesis and virulence gene expression. The data emphasize the intimate cross-regulation between metabolism and virulence in bacterial pathogens. 


\section{Introduction}

Listeria monocytogenes $(\mathrm{Lm})$ is a Gram-positive, facultative, intracellular pathogen and the causative agent of listeriosis, a disease that can lead to severe clinical manifestations in pregnant women, neonates and immunocompromised adults (1). Lm 60 is characterized by its intracellular lifestyle, but can also grow outside the host on soil 61 and vegetation, as well as on food products (2). In the mammalian host, $L m$ invades a 62 wide array of cells (phagocytic and non-phagocytic) by expressing specialized proteins, named internalins, that facilitate its internalization (e.g., InlA and InlB) (3). Upon internalization, the bacteria are initially found within a membrane-bound 65 vacuole, from which they escape into the host cell cytosol via the action of several 66 virulence factors - the pore-forming toxin Listeriolysin O (LLO, encoded by the hly 67 gene), and the two phospholipases PlcA and PlcB (4-6). In the host cell cytosol, the 68 bacteria utilize host-derived metabolites to support growth (7-10), and using ActA 69 protein hijack the host actin-polymerization machinery to move around the cell and 70 spread from cell to cell $(11,12)$. All of the above-mentioned virulence factors as well 71 as many others are positively regulated by PrfA, the master virulence regulator of $\mathrm{Lm}$ $(13,14)$.

Multiple metabolic and physiological signals are responsible for the transition of $\mathrm{Lm}$ from the saprophytic to the pathogenic state (15-20). Many of these signals converge 75 at PrfA, and directly or indirectly regulate its transcription, translation and activity. 76 One of these signals is low concentration of branched-chain amino acids (low 77 BCAA), a condition that is found in the intracellular niche. It was previously 78 demonstrated by our lab that $L m$ responds to low BCAA by upregulating the 79 expression of PrfA, which, in turn, activates the virulence genes (15). This response was shown to depend on the global metabolic regulator CodY, which is also a sensor of BCAA, as it directly binds isoleucine $(15,21,22)$. Under high-BCAA conditions, CodY was shown to bind isoleucine and in that form to repress the transcription of many metabolic genes, including those involved in BCAA biosynthesis (21-25). While this was considered the main mechanism of CodY regulation, we demonstrated 85 that in $L m$ CodY is also active under low BCAA conditions, i.e., in its isoleucineunbound form, where it up- and downregulates the transcription of many genes, among them prfA (up-regulating its transcription), hence playing a role in the 
expression is essentially linked to the availability of BCAA in the intracellular niche, 90 they further indicated that sensing of the metabolic host cell cytosol environment is 91 key to the regulation of $L m$ virulence.

Another metabolite that was recently shown to affect $\mathrm{Lm}$ virulence is glutathione.

Glutathione is a low-molecular-weight peptide thiol that is highly abundant in the host 95 cell cytosol in its reduced form (GSH), functioning as a redox buffer, antioxidant and 96 enzyme cofactor (26). Glutathione is also synthesized by some bacteria, mainly Gram97 negative and few Gram-positive (such as $L m$ ), and plays a role in redox homeostasis 98 and bacterial survival under oxidative stress (26). A previous study demonstrated that 99 Lm's glutathione synthetase, GshF, plays a critical role in the induction of the 100 virulence genes during infection (17). GshF is a bifunctional enzyme that catalyzes 101 the two reactions that synthesize the tripeptide glutathione (i.e., L- $\gamma$-glutamyl-Lcysteinylglycine) (27). It first ligates the $\gamma$-carboxyl group of L-glutamate to Lcysteine, a reaction that is the rate-limiting step of glutathione biosynthesis, and then condenses the product $\gamma$-glutamylcysteine with glycine. GSH itself was shown to 103 allosterically bind PrfA, and act as its activating cofactor $(28,29)$. More specifically, the binding of glutathione to PrfA was shown to cause a conformational change that primes its binding to DNA, as shown for other ligand-binding Crp/Fnr transcription regulators $(28,29)$.

As indicated, cysteine is one of the building blocks of glutathione, and its rate-limiting precursor, yet, it is not synthesized by $\mathrm{Lm}$. $\mathrm{Lm}$ lacks the ability to reduce sulfate to sulfide, which is required for cysteine biosynthesis, and hence has to import cysteine from the environment (30). Notably, $\mathrm{Lm}$ is also auxotrophic to methionine (which also has to be imported), and it cannot synthesize cysteine from methionine, as it lacks the transsulfuration pathway that converts methionine to cysteine $(9,30)$. To date, two transport systems have been shown to be involved in the acquisition of cysteine by $\mathrm{Lm}$. The first is the ABC-transporter Lmo0135-0137 (whose substrate binding protein is $\mathrm{CtaP}$ ), that was shown to support the uptake of free cysteine during growth in a synthetic medium (31). The other system is the OppABCDF transporter, that was shown to import oligopeptides, including cysteine-containing peptides, that were shown to serve as a source of cysteine for glutathione synthesis and PrfA activation 
(20). While these systems were reported to promote to $\mathrm{Lm}$ invasion and virulence gene expression in mammalian cells, respectively, their contribution to $\mathrm{Lm}$ intracellular growth was only partial $(20,31,32)$, implying that additional systems are involved in the acquisition of cysteine within the intracellular niche.

In this study, we report on TcyKLMN, another ABC-transporter that is directly involved in cysteine uptake in $\mathrm{Lm}$. We show that this transporter imports both cystine and cysteine and plays a role in the activation of $\mathrm{Lm}$ virulence gene expression by supplying cysteine for glutathione synthesis. Further, we demonstrate that this transporter is regulated by $\mathrm{CodY}, \mathrm{CymR}$ and CysK, three metabolic factors that act as repressors under nutrient-rich conditions. The findings presented here establish that cysteine import is key to the transition of $\mathrm{Lm}$ to the pathogenic state, and provide another example of the coupling of metabolism and virulence in bacterial pathogens.
123

124

125

126

127

128

129

130

131

132

133

134

135

136

137 


\section{Results}

Genes associated with cysteine uptake and metabolism modulate virulence gene expression

We previously performed a genetic screen of a mariner transposon mutant library in search of genes that differentially regulate the virulence genes of $\mathrm{Lm}$ strain 10403S under low BCAA. The screen identified multiple genes that were associated with cysteine uptake and metabolism: tcyN (LMRG_01497), ytmO (LMRG_01498), $L M R G \_01492$ and cysK (LMRG_02645) (Figure 1A and S1A) (33). Interestingly, the first three genes mapped to the $y t m I$ operon, which in B. subtilis was shown to encode the cystine ABC-transporter, TcyJKLMN (Figure 1A, of note, in Lm this operon lacks the tcyJ gene) (34). tcyN encodes the transporter's ATP-binding protein, whereas $y$ tmO and $L M R G \_01492$ encode a monooxygenase and an FMN reductase, respectively, whose functions in cystine uptake/metabolism remain elusive. Apart from this locus, the cys $K$ gene was also identified, encoding an $\mathrm{O}$-acetylserine thiollyase (which is part of the cysteine synthase complex), which plays a role in cysteine regulation and synthesis (Figure 1A and S1B). In B. subtilis, CysK was shown to be a trigger enzyme that acts both as a metabolic enzyme and a transcription regulator, the latter via interaction with CymR, a key transcription regulator in cysteine/methionine metabolism (35). It was shown that CymR and CysK form a complex that negatively regulates the $y t m I$ operon by repressing the transcription of its activator YtII (also called AscR (36, 37), which is encoded upstream to the operon in the opposite direction (a similar gene organization exists in Lm, Figure 1A) (38). Although ytlI and $\operatorname{cymR}$ were not identified in our screen, we found homologues in the $L m$ genome (LMRG_01491 and $L M R G \_01455$, respectively), suggesting that the Lm ytmI operon is regulated similarly to that of $B$. subtilis.

To investigate the cysteine-associated genes and their role in the regulation of $\mathrm{Lm}$ 
expresses the $\operatorname{lu} A A B C D E$ genes under the control of the PrfA-regulated hly promoter, which was cloned on the integrative plasmid pPL2 (pPL2-Phly-lux). Of note, wild type (WT) bacteria carrying this plasmid grown in a minimal defined medium containing low BCAA (low $\underline{B} C A A$ minimal medium, LBMM) display an enhanced luminescence profile, which represents the increased transcription of the hly gene [previously shown in (21)]. As mentioned, this upregulation of hly transcription in LBMM is completely dependent on CodY, as, under this condition, CodY activates the transcription of PrfA $(15,21)$. Examining the luminescence profiles of cys $K:: T n$ and $t c y N:: T n$ during growth in LBMM, we observed that the mutants differentially affect Phly-lux expression in comparison to WT bacteria. While the cysK::Tn mutant exhibited an enhanced luminescence profile, $t c y N:: T n$ failed to show luminescence 182 signals (Figure 1 B-C) (33). To validate these phenotypes, we generated clean deletion mutants of $c y s K$ and $t c y K$, the latter encoding the substrate binding protein 183 (SBP) of TcyKLMN (LMRG_01494). SBPs of ABC transporters (specifically 184 importers) are key factors that bind the transported substrate extracellularly and 185 facilitate its import into the cell, hence determining the substrate specificity of the 186 transporter (39). Since both $t c y K$ and $t c y N$ are essential components of TcyKLMN, we chose to delete $t c y K$ instead of $t c y N$, to further investigate the transporter's substrate specificity. As shown in Figure $1 \mathrm{D}-\mathrm{E}, \Delta c y s K$ and $\Delta t c y K$ recapitulated the phenotypes of their corresponding transposon mutants. Of note, the mutants grew similarly to WT bacteria in LBMM and in the rich medium BHI (Figures 1 D-E and Figure S3 A-B). Since in B. subtilis CysK was shown to repress tcyJKLMN by forming a complex with 187 188 189 190 191 192 CymR, we generated a $\triangle c y m R$ mutant of $L m$ and analyzed its luminescence profile 193 during growth in LBMM using the Phly-lux reporter system. The data indicated that 194 $\triangle \operatorname{cymR}$ behaves similarly to $\Delta c y s K$, i.e., exhibits an enhanced luminescence profile, 195 overall demonstrating that CysK and CymR negatively affect the transcription of hly 196 (Figure 1F and Figure S3C). To confirm the effects of TcyK, CysK and CymR on 197 the expression of the virulence genes, the transcription levels of actA, plcA and hly 198 (three major virulence genes of $\mathrm{Lm}$ ) were evaluated in $\Delta t c y K, \Delta c y s K$ and $\Delta c y m R$ 199 mutants and compared to those in WT bacteria grown in LBMM, using qRT-PCR. As 200 shown in Figure 1G, the transcription level of the virulence genes corroborated the 201 luminescence data, demonstrating a low transcription level in $\Delta t c y K(\sim 10$-fold) and an 202 enhanced transcription level in $\Delta c y s K$ and $\triangle c y m R$ ( $\sim 15$-fold) in comparison to WT 203 bacteria. These phenotypes were further complemented by introducing a plasmid 
containing a copy of $c y s K$ or $t c y K$ to the corresponding mutants, demonstrating WT

TcyKLMN is a cystine/cysteine transporter

The transport specificity of $\mathrm{ABC}$ transporters that functions as importers is dictated

almost exclusively by the binding specificity of their cognate substrate binding proteins (SBP). Therefore, to determine the substrate specificity of $L m$ TcyKLMN, near homogeneity (Figure S5). We then used isothermal titration calorimetry (ITC) to measure the binding of different substrates to TcyK. We found that TcyK binds cystine (CSSC, the reduced form of cysteine) with a dissociation constant $\left(K_{D}\right)$ of 13 $\mu \mathrm{M}$ and L-cysteine with a $K_{D}$ of $66.7 \mu \mathrm{M}$ (Figure 2A). We also tested TcyK for minimal defined medium containing either cysteine or CSSC as a sole source. Since display a growth disadvantage. Indeed, as shown in Figure 2B, when either CSSC or cysteine were added at limited concentrations $(<0.5 \mathrm{mM})$, the growth of $\Delta t c y K$ was significantly attenuated relative to WT bacteria. These experiments suggest that $\mathrm{Lm}$ TcyKLMN imports both CSSC and cysteine.

Since TcyKLMN was identified under low BCAA, we next investigated its expression tcyN and ytlI in WT bacteria grown in LBMM versus BHI. The results demonstrated that the transporter is highly expressed in LBMM ( 100-fold in comparison to BHI, shown by the transcription level of $t c y K$ and $t c y N$ ), and that the YtlI activator gene is also up-regulated ( 10-fold) (Figure 3B). Examining the ytmI-ytlI intergenic region, 
regulation of the YtII activator (Figure 3A). To test this hypothesis, we analyzed the transcription levels of $y t l I$ and $t c y K$ in WT and $\Delta \operatorname{cod} Y$ bacteria grown in BHI and LBMM, using qRT-PCR. The experiments confirmed that under nutrient-rich conditions (i.e., in BHI) CodY represses the ytlI gene, and consequently tcyK (Figure $3 \mathrm{C}$ ), and further demonstrated that this repression is relieved when BCAA are limited (i.e., in LBMM) (Figure 3D). We next investigated the role of CysK and CymR in the regulation of TcyKLMN (i.e., the $y t m I$ operon). Of note, since $L m$ does not assimilate sulfate to sulfide, we assumed that CysK acts as a CymR co-repressor and not as an O-acetylserine thiol-lyase, a function that requires sulfide (Figure S1B). As mentioned, in B. subtilis it was shown that CysK and CymR form a complex that represses the TcyJKLMN transporter genes (35). Based on the B. subtilis CymR 250 binding-motif (38), we identified two putative CymR binding-sites in the ytmI-ytlI 251 regulatory region of $\mathrm{Lm}$ : one upstream the $y t l I$ gene and the other upstream the $y \mathrm{tmI}$ 252 operon (Figure 3A). To examine the potential regulation of this gene locus by CymR 253 and CysK, we first analyzed the transcription levels of $y t l I$ and $t c y K$ in $\Delta c y m R$ and 254 $\triangle$ cys $K$ mutants in comparison to WT bacteria grown in BHI and LBMM. The data clearly demonstrated that CymR and CysK act as repressors, down-regulating the transcription of $y t l I$ and the $y t m I$ operon under both BHI and LBMM conditions 257 (Figure $3 \mathrm{C}-\mathrm{D}$ ). Since in B. subtilis it was shown that CysK and CymR respond to the 258 availability of cysteine, we repeated this experiment using a minimal defined medium 259 containing low concentration of cysteine (LCMM, containing $0.08 \mathrm{mM}$ cysteine). Interestingly, the data demonstrated that under these conditions, the repression of $y t l I$ 260 by CymR and CysK was fully relieved, whereas tcyK (representative of the 261 transporter genes) was still repressed by these proteins ( $\sim 15$-fold) (Figure 3E). These 262 findings established that TcyKLMN is negatively regulated, both directly and indirectly, by at least three factors: CodY, CymR and CysK, which collectively 264 respond to changes in BCAA and cysteine levels, and possibly to additional 265 metabolites that affect CymR and CysK. Taken together, the data indicated that under 266 nutrient-rich conditions, all three factors repress the ytmI operon, whereas under low 267 BCAA conditions, the operon is up-regulated, albeit, not to its full capacity, as CymR 268 and CysK still repress its transcription to some extent. These findings imply a complex regulation of TcyKLMN in response to different metabolic or environmental cues. 
In light of these observations, we next examined whether the increased expression of the virulence genes in $\triangle c y m R$ and $\triangle c y s K$ is due to the increased expression of TcyKLMN. For this purpose, we generated double deletion mutants, lacking either cysK or cymR and tcyK ( $\Delta c y s K / \Delta t c y K$ and $\Delta c y m R / \Delta t c y K)$ and analyzed their Phly-lux luminescence profiles during growth in LBMM. Notably, both mutants failed to activate Phly-lux expression, similarly to $\Delta t c y K$, indicating that TcyKLMN itself plays a role in the activation of the virulence genes under low BCAA (Figure 4 and Figure S3 F-G).

Cysteine import via TcyKLMN drives glutathione biosynthesis, which triggers 282 the induction of the virulence genes

Having discovered that in LBMM, TcyKLMN is required for the induction of the 284 virulence genes and not for bacterial growth (Figure 1E), we hypothesized that its 285 role in cysteine import may directly feed into glutathione biosynthesis, which, in turn, stimulates the activity of PrfA. This would account for the failure of all the tested 286 transporter mutants (i.e., $\Delta t c y K, t c y N:: T n, \Delta c y m R / \Delta t c y K$ and $\Delta c y s K / \Delta t c y K$ ) to express the virulence genes in LBMM. To test this hypothesis, we first constructed a mutant deleted of the glutathione synthase gene $(\Delta g s h F)$ and examined its impact on the activation of virulence genes in LBMM using the Phly-lux reporter system. As shown in Figure 5A, $\Delta g s h F$ displayed practically zero expression of the lux genes (similarly 287 to $\Delta t c y K$ ), however this phenotype was fully restored to WT levels by the exogenous addition of GSH $(20 \mathrm{mM})$. These observations indicated that glutathione biosynthesis 289 290 291 is absolutely required for the activation of the virulence genes in LBMM. Similarly, the transcription of virulence genes in $\Delta t c y K$ was restored by the addition of GSH; $\Delta t c y K$ bacteria supplemented with $20 \mathrm{mM}$ of GSH exhibited a luminescence profile 294 295 296 that was similar to that of WT bacteria, supporting the premise that TcyKLMN is 297 involved in glutathione biosynthesis (Figure 5B). To directly examine this hypothesis, we measured the total concentration of glutathione (including reduced and 299 oxidized forms, GSH + GSSG) in $\Delta t c y K$ bacteria in comparison to WT $L m$, using 300 $\Delta g s h F$ as a control. Remarkably, the internal glutathione concentration of $\Delta t c y K$ was 301 as low as that measured in $\Delta g s h F$, below the detection level of the glutathione assay 302 kit (Figure 5C), indicating that TcyKLMN plays a role in glutathione biosynthesis, 303 most likely by supplying the rate-limiting precursor, cysteine. 
In light of these findings, we next examined whether the enhanced expression of the virulence genes in $\triangle c y s K$ and $\triangle c y m R$ is a result of increased production of 307 glutathione, as these strains highly express TcyKLMN, and hence presumably import 308 more cysteine for glutathione synthesis. Notably, internal measurements of 309 glutathione in $\triangle c y s K$ and $\triangle c y m R$ bacteria demonstrated a 2-fold increase in the amount of glutathione in comparison to WT bacteria, supporting the hypothesis that higher cysteine uptake can increase glutathione biosynthesis (Figure 5C). Moreover, these observations demonstrated that cysteine import by TcyKLMN feeds into glutathione biosynthesis and further confirmed that cysteine is indeed a rate-limiting substrate of GshF. To evaluate whether the enhanced virulence gene expression in $\triangle \operatorname{cys} K$ and $\triangle c y m R$ relies on glutathione biosynthesis, we combined these mutants with a deletion of $g s h F$ (generating the double deletion mutants: $\Delta c y s K / \Delta g s h F$ and $\Delta c y m R / \Delta g s h$ ), and examined virulence gene expression in LBMM, using the lux reporter system. As shown in Figure 5D, these mutants failed to express the lux genes, confirming that glutathione biosynthesis is absolutely required for virulence gene expression. Of note, these mutants, as well as $\Delta g s h$, grew like WT bacteria both in BHI and LBMM, overall demonstrating that glutathione itself is not essential for growth under these conditions (Figure 5A, D and Figure S3 H-J). Finally, we examined whether CymR, CysK and CodY regulate the transcription of $g s h F$ in BHI and LBMM, and found that they do not (Figure S6), supporting the conclusion that they affect glutathione biosynthesis by regulating the import of cysteine via TcyKLMN. We also examined the possibility that TcyKLMN imports glutathione, and found that it does not, as evident from ITC experiments using TcyK, and growth experiments using glutathione as a source of cysteine (Figure S7 A-B). Of note, these experiments clearly demonstrated that $\mathrm{Lm}$ can use exogenous GSH as a sole source of cysteine (Figure S7B). Altogether, these findings demonstrated that when grown in LBMM, TcyKLMN is the main transport system that supplies cysteine for glutathione biosynthesis, which, in turn, drives the expression of the virulence genes via the activation of PrfA.

\section{0}

311 
Finally, given the identified role of TcyKLMN in glutathione biosynthesis, we sought to investigate its impact on $\mathrm{Lm}$ intracellular growth and virulence gene expression in macrophage cells. We first analyzed the transcription level of $t c y K, t c y N$ and $y t l I$ in WT bacteria grown intracellularly in macrophage cells in comparison to bacteria grown in BHI. The data indicated a modest induction of TcyKLMN and its activator YtlI within the intracellular niche ( 3-fold) (Figure 6A). We next evaluated the expression of the virulence gene $p l c A$ in $\Delta t c y K$ and WT bacteria grown intracellularly 345 in macrophage cells, using $\Delta g s h F$ as a control. For this purpose, we used a 346 fluorescence-based reporter system that we previously constructed, which relies on 347 the expression of three consecutive $y f p$ genes under the regulation of the $p l c A$ 348 promoter (cloned in the integrative plasmid pPL2, pPL2-PplcA-3yfp) (33). Using this 349 system, we observed a $\sim 40 \%$ reduction in the expression of plcA in $\Delta t c y K$ in comparison to WT bacteria, whereas a more dramatic reduction of $\sim 75 \%$ was observed in $\Delta g s h F$ (Figure 6B). While these findings demonstrate that TcyKLMN contributes to the induction of the virulence genes during macrophage cell infection, they implied that it is not the sole supplier of cysteine within the intracellular niche, corroborating previous reports demonstrating that $L m$ also utilizes oligopeptides as a source of amino acids, including cysteine, which are imported by the Opp transporter $(20,41)$. We next examined the intracellular growth of $\Delta t c y K$ and $\Delta g s h F$ in macrophage cells in comparison to WT bacteria. Notably, the data demonstrated a late growth defect for $\Delta t c y K$ that was apparent at $6 \mathrm{~h}$ post-infection (Figure 6C). 350 351 352 353 354 355 356 357 Interestingly, a similar phenotype was reported for the Opp transporter deletion 359 mutant $(\triangle o p p D F)$, which also exhibited an intracellular growth defect at 6 to $7 \mathrm{~h}$ post360 infection of macrophage cells (20). Evaluating $\Delta g s h F$ intracellular growth in 361 macrophage cells, we observed a more profound growth defect, starting at $3 \mathrm{~h}$ postinfection, indicating that glutathione biosynthesis by $\mathrm{Lm}$ is critical for intracellular growth, and hence has to be accompanied by cysteine uptake (Figure 6D). Taken together, the results presented here support the premise that $L m$ uses multiple 362 363 364 365 transport systems to acquire cysteine in the intracellular niche, among them TcyKLMN. 


\section{Discussion}

This study is a direct continuation of our previous reports demonstrating that low

availability of BCAA is sensed by $L m$ as a signal to activate the expression of

virulence genes. While we established that the metabolic regulator $\operatorname{CodY}$ is repressed under nutrient-rich conditions, and up-regulated when BCAA are limited.

This repression was linked to $\operatorname{CodY}$, which we found to repress the activator of the 391

ytmI operon, YtlI. As indicated, low BCAA is the same condition at which PrfA

An important observation made in this study was that glutathione levels are largely 
activation of PrfA. In a way, these findings uncover a new pathway by which $\mathrm{Lm}$ virulence can be regulated in the mammalian host responding to changes in cysteine availability. CodY, CymR and CysK were also found to regulate the expression of TcyKLMN, and hence to modulate the expression of the virulence genes. These regulators still repressed TcyKLMN under conditions of low concentrations of BCAA and cysteine, implying that other metabolic or environmental signals may be involved. In $B$. subtilis, it was shown that the $y t m I$ operon is differentially regulated under disulfide stress and changes in sulfur availability, hence it is possible that the $L m$ CymR and CysK respond to these conditions $(36,38)$. Moreover, since glutathione plays a role in redox conditions, it is possible that CymR and CysK respond to these conditions as well. In this respect, it was demonstrated in Staphylococcus aureus that CymR senses oxidative stress via thiolation of its cysteine residue (Cys-25), and hence the YtmI operon is up-regulated under oxidizing environments (43). Taken together, these reports indicate that the YtmI operon is regulated by multiple metabolic and environmental cues. Interestingly, the $y t m I$ operon of $B$. subtilis is independent of CodY regulation, as evident from RNA-seq experiments and genomewide analyses of CodY binding sites $(44,45)$. It is tempting to speculate that CodY regulation of Lm's TcyKLMN evolved to cope with the pathogenic lifestyle of this bacterium, yet $L m$ and B. subtilis differ greatly in their sulfur, cysteine and methionine metabolism, which can lead to differences in the regulation of these pathways. Notwithstanding, this study revealed an intriguing link between CodY regulation, cysteine import and glutathione biosynthesis in $\mathrm{Lm}$, portraying additional mechanism to control the expression of the virulence genes.

Here, we showed that the SBP of TcyKLMN (i.e., TcyK) specifically binds cysteine, and to a better extent, CSSC, suggesting that it primes the import of these two related substrates. CSSC is the oxidized form of cysteine, containing two cysteine molecules that are linked via a disulfide bond, and hence is expected to be more prevalent in oxidizing environments, such as within phagosomes. Outside the mammalian cells secreted by the liver), yet it is quickly oxidized and imported into the cells via specialized CSSC transporters, e.g. the CSSC/glutamate antiporter, cXT, which also plays a role in glutathione synthesis (46-49). Interestingly, within the cells, 
cysteine from methionine, using the transsulfuration pathway, or alternatively break down glutathione to salvage cysteine (46). In respect to $\mathrm{Lm}$ 's intracellular lifestyle, it is likely that it encounters CSSC within the phagosome/vacuole environment. It is possible that TcyKLMN plays a role during cell invasion or during spread from cell to cell (i.e., in the primary and secondary vacuoles, respectively), or when $\mathrm{Lm}$ switches to persistent infection, i.e., when it resides in lysosome-like vacuoles for long periods $(50,51)$. While we did not identify a phenotype for $\Delta t c y K$ upon mice infections (data not shown), we observed a late intracellular growth defect during infection of bone 446 marrow-derived macrophage cells. These experiments further demonstrated a reduced 447 transcription level of $p l c A$ in $\Delta t c y K$, supporting the premise that TcyKLMN 448 contributes to the activation of PrfA during $\mathrm{Lm}$ infection of mammalian cells. Since CSSC and cysteine are relatively limited in the intracellular environment, it is not 450 surprising that $\mathrm{Lm}$ acquired multiple means to scavenge this essential amino acid. As mentioned, $L m$ exploits peptides as a source of amino acids, and the Opp transporter was shown to import cysteine-containing peptides as a source of cysteine for glutathione biosynthesis $(20,41)$. The finding that $\Delta t c y K$ exhibits a $40 \%$ reduction in $p l c A$ transcription, as compared to the $75 \%$ reduction in $\Delta g s h F$, supports the premise that $L m$ exploits multiple systems to scavenge cysteine.

Reniere et al. suggested that $L m$ also exploits host glutathione (GSH) to activate PrfA 453 454 (17). However, this mode of PrfA activation appeared to be minor, as $\Delta g s h F$ bacteria hardly activated the virulence genes in the intracellular environment (17), overall indicating that host-derived GSH cannot replace the glutathione produced by $\mathrm{Lm}$. In this regard, it remains an open question why $L m$ synthesizes GSH and uses it as an intracellular signal, considering its high abundance in the intracellular niche, much more than cysteine. Other bacterial pathogens, such as Burkholderia pseudomallei and Francisella tularensis were shown to exploit host GSH as an activating signal of virulence gene expression and as a source of cysteine, respectively (52, 53). capable of importing GSH and uses it both to activate PrfA, and as a source of cysteine (Figure 5 and S7B) (54). While these findings imply that $\mathrm{Lm}$ encodes a glutathione transporter, or, alternatively, uses a nonspecific system such as di/tripeptide transporters to import glutathione, it is not clear why it does not use them in 
transporter DppBCDF was shown to import glutathione, using the SBP protein GbpA

(55). It is of particular interest to decipher the structural and molecular mechanism by

which glutathione is imported in Gram-positive bacteria, especially intracellular

mutans, it was shown that the CSSC ABC-transporter, TcyBC (not found in Lm), imports both CSSC and glutathione using two distinct SPB proteins (55). The SBP that binds glutathione (GshT) was found to be encoded elsewhere on the bacterial chromosome, and not near the $t c y B C$ genes. While this phenomenon of a shared 480 permease is not new, the discovery that $S$. mutans holds a distinct SBP that primes glutathione import via another transporter was new, providing early insights into 482 glutathione import in Gram-positive bacteria (56). Interestingly, we found that TcyK 483 shares 30\% amino-acid sequence identity with the S. mutans GshT, yet our data 484 indicated that it does not bind glutathione.

In summary, this study demonstrated that cysteine import is critical for virulence gene 487 expression in $\mathrm{Lm}$. The data imply that multiple metabolic and environmental cues are 488 involved in the regulation of cysteine import and hence affect glutathione 489 biosynthesis, placing this function at the heart of $\mathrm{Lm}$ patho-metabolism. As different 490 bacterial pathogens acquired different mechanisms to sense the mammalian niche, it is 491 interesting to learn how they exploit host and bacterial metabolites as signals and 492 effectors of virulence. 


\section{Materials and Methods}

\section{Ethics statement}

Experimental protocols were approved by the Tel Aviv University Animal Care and Use Committee (01-15-052, 04-13-039) and were in accordance with the Israel Welfare Law (1994) and the National Research Council guide (Guide for the Care and Use of Laboratory Animals 2010).

\section{Bacterial strains, plasmids and primers}

Listeria monocytogenes $(\mathrm{Lm})$ strain $10403 \mathrm{~S}$ was used as the WT strain and as the parental strain to generate allelic exchange mutant strains (Table S1). E. coli XL-1 blue strain (Stratagene) was used to generate vectors and E. coli SM-10 strain (57) was used for plasmid conjugation to Lm. Plasmids and primers used in this study are listed in Table $\mathbf{S} 1$ and Table $\mathbf{S 2}$, respectively.

\section{Growth conditions}

$\mathrm{Lm}$ bacteria were grown at $37^{\circ} \mathrm{C}$, with agitation, in brain heart infusion (BHI), as a 511 nutrient-rich medium, or in minimal defined medium (MM). MM (phosphate buffer $48.2 \mathrm{mM} \mathrm{KH}_{2} \mathrm{PO}_{4}$ and $1.12 \mathrm{M} \mathrm{Na}_{2} \mathrm{HPO}_{4}, \mathrm{pH}$ 7, $0.41 \mathrm{mg} / \mathrm{ml}$ magnesium sulfate, 10 512 513 $\mathrm{mg} / \mathrm{ml}$ glucose, $100 \mu \mathrm{g} / \mathrm{ml}$ of each amino acid (methionine, arginine, histidine, 514 tryptophan, phenylalanine, cysteine, isoleucine, leucine and valine), $600 \mu \mathrm{g} / \mathrm{ml}$ 515 glutamine, $0.5 \mathrm{mg} / \mathrm{ml}$ biotin, $0.5 \mathrm{mg} / \mathrm{ml}$ riboflavin, $20 \mathrm{mg} / \mathrm{ml}$ ferric citrate, $1 \mathrm{mg} / \mathrm{ml}$ 516 para-aminobenzoic acid, $5 \mathrm{ng} / \mathrm{ml}$ lipoic acid, $2.5 \mathrm{mg} / \mathrm{ml}$ adenine, $1 \mathrm{mg} / \mathrm{ml}$ thiamine, 1 517 $\mathrm{mg} / \mathrm{ml}$ pyridoxal, $1 \mathrm{mg} / \mathrm{ml}$ calcium pantothenate and $1 \mathrm{mg} / \mathrm{ml}$ nicotinamine) was 518 prepared as described previously (58). For analysis of auxotrophies, Lm was grown 519 with $0-2000 \mu \mathrm{g} / \mathrm{ml}$ of either cysteine or methionine or neither, as indicated. For growth under low BCAA, MM was freshly made with 10-fold less isoleucine, leucine 520 521 and valine (resulting in a final concentration of $10 \mu \mathrm{g} / \mathrm{ml}$ ) was named low-BCAA 522 minimal defined medium (LBMM). For growth under limited concentrations of cysteine, MM was freshly made with 10-fold less cysteine (resulting in a final 523 524 concentration of $10 \mu \mathrm{g} / \mathrm{ml}$ ). For growth in glutathione medium, $20 \mathrm{mM}$ reduced glutathione (GSH) were freshly added to LBMM. For growth with cysteine or with 
either cystine (CSSC), reduced or oxidized glutathione (GSH or GSSG, respectively)

\section{Lux reporter assay}

Overnight BHI cultures harboring a Phly-lux luciferase reporter system (pPL2-Phly532 luxABCDE) (59) were washed 3 times with PBS, adjusted to $\mathrm{OD}_{600}=0.03$ in fresh growth $\left(\mathrm{OD}_{600}\right)$ were measured every 15 min after shaking for $1 \mathrm{~min}$, using a Synergy

\section{Bacterial RNA extraction and qRT-PCR}

Bacteria grown in the indicated medium were harvested at mid-logarithmic growth 539 $\left(\mathrm{OD}_{600}\right.$ of $\left.\sim 0.3\right)$. Total RNA was extracted from bacteria using the RNAsnap method 540 (60). Briefly, bacterial pellets were washed with AE buffer (50 mM NaOAc pH 5.2, $10 \mathrm{mM}$ EDTA) and then resuspended in 95\% formamide, $18 \mathrm{mM}$ EDTA, $1 \%$ 2mercaptoethanol and $0.025 \%$ SDS. Bacterial lysis was performed by vortexing extracts with $100 \mu \mathrm{m}$ zirconia beads (OPS Diagnostics), followed by incubation at 544 $95^{\circ} \mathrm{C}$. Nucleic acids were precipitated with ethanol and treated with Turbo-DNase (Ambion), followed by standard phenol extraction. Total RNA $(1 \mu \mathrm{g})$ was reverse545 transcribed to cDNA using qScript (Quanta). qRT-PCR was performed on $10 \mathrm{ng}$ cDNA using FastStart Universal SYBR Green Master (Roche) in a StepOne Plus realtime PCR system (Applied Biosystems). The transcription level of each gene was normalized to that of the reference gene $r p o D$.

Putative binding sites of transcriptional regulators were identified using the MAST 
Transcription start sites (TSS) were either previously identified (64) or manually 556 predicted based on the promoter sequence.

Intracellular growth in macrophage cells

Bone marrow-derived macrophages (BMDM) were used for $L m$ infection 560 experiments. The cells were isolated from 8 week-old female C57BL/6 mice (Envigo, Israel) and cultured in BMDM medium (Dulbecco's modified Eagle medium (DMEM) supplemented with $20 \%$ fetal bovine serum, sodium pyruvate $(1 \mathrm{mM})$, L563 glutamine $(2 \mathrm{mM}), \beta$-mercaptoethanol $(0.05 \mathrm{mM})$ and monocyte-colony stimulating 564 factor (M-CSF, L929-conditioned medium), as described previously (65)). BMDM cells $\left(2 \times 10^{6}\right)$ were seeded in a $60 \mathrm{~mm}$ Petri dish, on glass coverslips, in $5 \mathrm{ml}$ BMDM 565 medium and incubated overnight (O.N.) in a $37^{\circ} \mathrm{C}, 5 \% \mathrm{CO}_{2}$ forced-air incubator. $\mathrm{Lm}$ 566 bacteria $\left(8 \times 10^{6}\right)$ grown O.N. at $30^{\circ} \mathrm{C}$ without agitation, were used to infect BMDM 567 cells (MOI of 1). Thirty minutes post-infection, macrophage monolayers were washed 568 and fresh medium was added. Gentamicin was added $1 \mathrm{~h}$ post-infection to a final 569 concentration of $5 \mu \mathrm{g} / \mathrm{ml}$ in order to limit the growth of extracellular bacteria. At each 570 time point, three coverslips were transferred to $2 \mathrm{ml}$ sterile water to release the 571 intracellular bacteria. Serial dilutions of the resulting lysate were plated on BHI agar plates and the CFUs were counted after $24 \mathrm{~h}$ incubation at $37^{\circ} \mathrm{C}$.

WT and mutant strains expressing three consecutive YFP proteins under the 577 regulation of the $p l c A$ gene promoter (cloned on the pPL2 integrative plasmid) were 578 used to infect BMDM on $20 \mathrm{~mm}$ slides. Three hours post-infection, cells were fixed with $4 \% \mathrm{v} / \mathrm{v}$ paraformaldehyde-PBS and permeabilized with $0.05 \% \mathrm{v} / \mathrm{v}$ Triton X-100. 580 DNA was stained with DAPI-containing Vectashield mounting medium (Vector 581 laboratories inc.). Fluorescent images were captured using a Zeiss LSM 510-META 582 confocal microscope. 
RNA was purified from WT $L m$ bacteria intracellularly grown in J774A.1 586 macrophage cells, as previously described for BMDM macrophages (66). Briefly, 587 three $145 \mathrm{~mm}$ dishes were seeded with $2 \times 10^{7}$ cells that were then infected in parallel 588 with $2 \times 10^{9}$ bacteria. Thirty minutes post-infection, cell monolayers were washed twice with PBS to remove unattached bacteria, and fresh medium was added. At $1 \mathrm{~h}$ 589 post-infection (h.p.i.), gentamicin $(50 \mu \mathrm{g} / \mathrm{ml})$ was added to limit extracellular bacterial 590 growth. At 6 h.p.i., the macrophages were lysed with $20 \mathrm{~mL}$ cold water, and cell 591 debris and nuclei were removed by centrifugation $\left(800 \mathrm{xg}, 3 \mathrm{~min}, 4^{\circ} \mathrm{C}\right)$. Released bacteria were quickly collected on $0.45 \mu \mathrm{m}$ filter membranes (Millipore) using a vacuum apparatus and snap-frozen in liquid nitrogen. Bacteria were recovered from the filters by vortexing the membranes in AE buffer $(50 \mathrm{mM}$ sodium acetate $\mathrm{pH} 5.2$, $10 \mathrm{mM}$ EDTA), and bacterial nucleic acids were extracted using the RNAsnap method (60), followed by ethanol precipitation. RNeasy Mini Kit DNase on column (QIAGEN) was used for DNase treatment.

\section{Cloning, overexpression and purification of TcyK}

600

Lm 10403S tcyK, encoding the TcyKLMN substrate binding protein, was synthesized and adjusted to the E. coli codon usage (Genescript). The gene was cloned into the 601 pET-19b vector (Novagen) for expression with an N-terminal His-tag. His-tagged TcyK was overexpressed in E. coli BL21-Gold (DE3) (Stratagene) cultured in Terrific Broth (TB) and induced at mid-log phase by addition of $1 \mathrm{mM}$ isopropyl b- D-1thiogalactopyranoside (IPTG; $1.5 \mathrm{~h}, 37^{\circ} \mathrm{C}$ ). Cells were harvested by centrifugation $\left(13,600 \times \mathrm{g}, 20 \mathrm{~min}, 4^{\circ} \mathrm{C}\right)$ and the pellet was stored at $-80^{\circ} \mathrm{C}$ until use. For purification, cells were homogenized in $50 \mathrm{mM}$ Tris- $\mathrm{HCl} \mathrm{pH} \mathrm{8,} 500 \mathrm{mM} \mathrm{NaCl}$, complete EDTA-free protease inhibitor (Roche), $30 \mathrm{mg}$ ml-1 DNase (Worthington), and $1 \mathrm{mM} \mathrm{MgCl}_{2}$. The cells were then ruptured by three passages through an EmulsiFlex-C3 homogenizer (Avestin), and the lysate was centrifuged at 34,500 $\times \mathrm{g}$ 602 603 604 605 606 607 608 for $30 \mathrm{~min}$, at $4^{\circ} \mathrm{C}$. The supernatant was loaded onto a nickel affinity column (HisTrap HP, GE Healthcare) on an AKTA Avant instrument. The protein was eluted using an imidazole gradient, and imidazole was removed from the sample by desalting (HiPrep 26/10, GE Healthcare). Protein purification was monitored by Coomassie staining of SDS-PAGE and size exclusion chromatography (Superdex 75 10/300 GL, GE 609 610 611 


\section{Isothermal titration calorimetry experiments}

Calorimetric measurements were performed with the Microcal iTC200 System (GE

Healthcare). Prior to measurements, purified TcyK was dialyzed against three

\section{Glutathione quantification}

Total glutathione (GSH+GSSG) concentration in bacteria was measured as described 634 previously (54). Briefly, bacteria were grown to mid-log phase in LBMM, 635 resuspended in PBS containing $1 \mathrm{mM}$ EDTA, pH 6.5, and lysed by vortexing with 636 $100 \mu \mathrm{m}$ zirconia beads (OPS Diagnostics). Cold $\left(4^{\circ} \mathrm{C}\right)$ lysates were deproteinated with an equal volume of metaphosphoric acid and quantified with a commercial kit

\section{Acknowledgments}

We thank Lior Lobel for critical review of the manuscript. This work was supported 
bioRxiv preprint doi: https://doi.org/10.1101/2021.09.07.459368; this version posted September 8, 2021. The copyright holder for this preprint (which was not certified by peer review) is the author/funder. All rights reserved. No reuse allowed without permission. 


\section{Figure Legends}

Fig 1. The TcyKLMN transporter and the regulatory factors CysK and CymR representation of the $y \mathrm{tmI}$ operon and the genes identified in Friedman et al. 2017. and OD 600nm measurements of bacteria grown in minimal defined media 656 supplemented with low concentrations of branched chain amino acids (LBMM). 657 Relative Luminescence Units (RLU) represent luminescence values normalized to the 658 respective OD 600nm value. OD 600nm values represent growth. The data represent 3 659 biological replicates. Error bars indicate standard deviation. (G) qRT-PCR analysis of 660 $a c t A, p l c A$ and hly transcription levels in indicated bacteria grown in LBMM. mRNA 661 levels were normalized to $r p o D$ mRNA and are represented as relative quantity (RQ), 662 relative to mRNA level in WT bacteria. The data represent 3 biological replicates. 663 Error bars indicate standard deviation. Asterisks represent $\mathrm{P}$-values $\left(*=\mathrm{P}<0.05,{ }^{*}=\right.$ 664 $\mathrm{P}<0.01, * * *=\mathrm{P}<0.001$, n.s. $=$ non- significant $)$ calculated by Student's $\mathrm{t}$-test. $\mathrm{P}$ 665 values represent a comparison to the BHI sample.

Fig 2. TcyKLMN is a cystine/cysteine transporter. (A) Isothermal Titration 668 Calorimetry (ITC) analysis, showing binding of TcyK to cystine (CSSC) or cysteine 669 (Cys). Shown are the consecutive injections of $2 \mu \mathrm{L}$ aliquots from a $200 \mu \mathrm{M}$ 670 Cys/CSSC solution into $200 \mu \mathrm{L}$ of $20 \mu \mathrm{M}$ TcyK. TCEP was used as a reducing agent when indicated. The upper panels show the calorimetric titration and the lower panels display the integrated injection heat derived from the titrations, for which the best-fit curve was used to calculate the $K_{D}$. The experiments were repeated independently 3 times, and the $K_{D}$ value is presented as a mean \pm standard deviation of these 3 experiments. (B) Growth of WT $L m$ and $\Delta t c y K$ bacteria grown in minimal defined 676 media supplemented with different concentrations of cysteine (Cys) or cystine 677 (CSSC) as a sole cysteine source. The data is presented as maximal OD 600nm values, corresponds to bacterial yield. The data represent 2 biological replicates. The experiment was repeated independently twice, with a total of 4 biological replicates. 680 Error bars indicate standard deviation. 
CymR. (A) A schematic representation of the ytmI-ytlI regulatory region. Putative and -35) were identified using BPROM. TSS denotes the transcription start sites, 687 either identified by Wurtzel et al., 2012, or predicted based on the promoter sequence. in rich media (BHI) and in LBMM. mRNA levels were normalized to $r p o D$ mRNA 690 and are represented as relative quantity (RQ), relative to mRNA level in WT bacteria 691 grown in BHI. The data represent 3 biological replicates. Error bars indicate standard 692 deviation. Asterisks represent $\mathrm{P}$-values $(*=\mathrm{P}<0.05, * *=\mathrm{P}<0.01$, $* * *=\mathrm{P}<0.001$, n.s. 693 = non- significant) calculated by Student's t-test. P-values represent a comparison to 694 the BHI sample. (C-E) qRT-PCR analysis of tcyK and ytlI transcription level in the indicated mutants grown in rich media (BHI) and minimal defined media supplemented with low concentration of either branched chain amino acids (LBMM) 695 696 or cysteine (LCMM). mRNA levels were normalized to rpoD mRNA and are 697 represented as relative quantity (RQ), relative to mRNA level in WT bacteria grown 698 in the indicated medium. The data represent 3 biological replicates. Error bars indicate 699 standard deviation. Asterisks represent $\mathrm{P}$-values $(*=\mathrm{P}<0.05, * *=\mathrm{P}<0.01, * * *=$ $\mathrm{P}<0.001$, n.s. $=$ non- significant) calculated by Student's t-test. P-values represent a comparison to the respective WT sample, unless indicated otherwise.

700 701 702 703 704

Fig 4. Enhanced expression of virulence genes in $\triangle$ cymR and $\triangle$ cysK is TcyKLMN 705

dependent. Luminescence of pPL2 Phly-lux reporter system (left) and growth (right) 706 of $\Delta c y s K / \Delta t c y K$ and $\Delta c y m R / \Delta t c y K$ bacteria grown in minimal defined media 707 supplemented with low concentration of branched chain amino acids (LBMM). 708 Relative Luminescence Units (RLU) represent luminescence values normalized to the 709 respective OD 600nm value. OD 600nm values represent growth. The data represent 3 710 biological replicates. Error bars indicate standard deviation. and $\triangle t c y K$ bacteria grown in minimal defined media with low concentration of 
to LBMM when indicated. Relative Luminescence Units (RLU) represent

luminescence values normalized to the respective OD $600 \mathrm{~nm}$ value. OD $600 \mathrm{~nm}$

standard deviation. Asterisks represent $\mathrm{P}$-values $(* * *=\mathrm{P}<0.001)$ calculated by

Luminescence of pPL2 Phly-lux reporter system (left) and growth (right) of

Fig 6. The TcyKLMN transporter promotes $\mathrm{Lm}$ intracellular growth in

macrophage cells. (A) qRT-PCR analysis of $t c y K, t c y N$ and $y t l I$ transcription level in 730

WT bacteria grown in rich media (BHI) or intracellularly in J774A.1 macrophage cell 731 line (IC). mRNA levels were normalized to $r p o D$ mRNA and are represented as relative quantity (RQ), relative to mRNA level in in the BHI sample. The data represent 3 biological replicates. Error bars indicate standard deviation. Asterisks 734 represent $\mathrm{P}$-values $(*=\mathrm{P}<0.05, * *=\mathrm{P}<0.01, * * *=\mathrm{P}<0.001$, n.s. $=$ non- significant $)$ calculated by Student's t-test. P-values represent a comparison to the BHI sample. (B) 736 Intracellular expression of $p l c A$ as indicated by the fluorescence of YFP proteins 737 expressed under the control of $p l c A$ promoter (pPL2-PplcA-3yfp) in WT Lm and 738 indicated mutants, grown in bone marrow derived macrophages (BMDM). 739 Fluorescence was measured 3 hours post infection using a florescence microscope.

The data represent at least 3 biological replicates. Error bars indicate standard 741 deviation. Asterisks represent $\mathrm{P}$-values $(*=\mathrm{P}<0.05, * *=\mathrm{P}<0.01$, $* * *=\mathrm{P}<0.001$, n.s. = non- significant) calculated by Student's t-test. P-values represent a comparison to the WT sample. (C-D) Intracellular growth of $\Delta t c y K$ and $\Delta g s h F$ mutants in BMDM 
Fig S1. Cysteine uptake and metabolic genes identified in a screen by Friedman 755

et al. (A) Phenotypes of transposon mutants as identified in Friedman et al., 2017. 756 WT bacteria and indicated transposon mutants were grown in minimal defined 757 medium supplemented with low concentration of branched chain amino acids 758 (LBMM). Relative Luminescence Units (RLU) represent luminescence values 759 normalized to the respective OD 600nm value. (B) Schematic representation of 760 cysteine transport via TcyKLMN and cysteine-glutathione biosynthesis. The genes 761 identified in the genetic screen are marked in red. OAS=O-acetylserine.

Fig S2. Lm strain 10403S is auxotrophic to cysteine and methionine. (A-B) 764 Growth of WT Lm strain 10403S in minimal defined media supplemented with 765 different concentrations of either cysteine or methionine. The data is presented as 766 maximal OD $600 \mathrm{~nm}$ values, corresponding to bacterial yield. The data represent 3 767 biological replicates. Error bars indicate standard deviation. (C) Growth of WT Lm in 768 minimal defined media without cysteine supplemented either with $1000 \mu \mathrm{g} / \mathrm{ml}, 2000$ 769 $\mu \mathrm{g} / \mathrm{ml}$ or no methionine. Growth of WT $\mathrm{Lm}$ in minimal defined media supplemented 770 with standard concentrations of cysteine $(100 \mu \mathrm{g} / \mathrm{ml})$ is shown as a control. OD 600 771 $\mathrm{nm}$ values represent growth. The data represent 3 biological replicates. Error bars 772 indicate standard deviation. (D) Growth of WT Lm in minimal defined medium 773 without methionine, supplemented with either $1000 \mu \mathrm{g} / \mathrm{ml}, 2000 \mu \mathrm{g} / \mathrm{ml}$ or no cysteine. 774 Growth of WT Lm in minimal defined media supplemented with standard 775 concentrations of methionine $(100 \mu \mathrm{g} / \mathrm{ml})$ is shown as a control. OD $600 \mathrm{~nm}$ values 776 represent growth. The data represent 3 biological replicates. Error bars indicate standard deviation.

Fig S3. Growth of deletion mutants generated in this study in the rich medium 780

BHI. WT bacteria and indicated strains (A-J) were grown in BHI. OD 600nm values 781 represent growth. The data represent 3 biological replicates. Error bars indicate 782 standard deviation. 
Fig S4. Complementation experiments of $\Delta t c y K$ and $\Delta c y s K$ mutants. qRT-PCR respective complementation strains harboring a copy of $t c y K$ or $c y s K$ gene under the tet promoter on pPL2 plasmid, grown in LBMM. mRNA levels were normalized to $r p o D$ mRNA and are represented as relative quantity (RQ), relative to mRNA level in 788 WT bacteria. The data represent at least 2 biological replicates. Error bars indicate 789 standard deviation. Asterisks represent $\mathrm{P}$-values $(*=\mathrm{P}<0.05, * *=\mathrm{P}<0.01, * * *=$ $\mathrm{P}<0.001$, n.s. = non- significant) calculated by Student's t-test. P-values represent a comparison to the WT sample unless indicated otherwise.

Fig S5. Purification of a short variant of TcyK. (A) Coomassie staining of SDS795 PAGE of Ni-NTA affinity purification of a His-tagged short TcyK (amino acids 38286). Lane 1: molecular weight marker (in $\mathrm{kDa}$ ), Lane 2: total protein extract, Lane 3: column-unbound fraction, Lane 4: wash with $60 \mathrm{mM}$ of imidazole, Lanes 5-11: fractions eluted using a linear gradient of 60 to $250 \mathrm{mM}$ imidazole. (B) Analysis of the purified protein by size exclusion chromatography. $80 \mu \mathrm{l}$ of a $0.8 \mathrm{mg} / \mathrm{ml}$ protein solution were injected on a Superdex 75 10/300 GL column (GE Healthcare).

Fig S6. gshF is not transcriptionally regulated by CymR, CysK and CodY. qRT803 PCR analysis of $g s h F$ transcription level in the indicated mutants grown in rich media 804 (BHI) and minimal defined media supplemented with low concentration of either 805 branched chain amino acids (LBMM). mRNA levels were normalized to rpoD mRNA 806 and are represented as relative quantity (RQ), relative to mRNA level in WT bacteria 807 grown in the indicated medium. The data represent at least 2 biological replicates. 808 Error bars indicate standard deviation Asterisks represent $\mathrm{P}$-values $(*=\mathrm{P}<0.05, * *=$ $\mathrm{P}<0.01, * * *=\mathrm{P}<0.001$, n.s. $=$ non- significant) calculated by Student's t-test. $\mathrm{P}$ 809 values represent a comparison to the respective WT sample.

Fig S7. The TcyKLMN transporter does not transport glutathione. (A) Isothermal 
integrated injection heat derived from the titrations. The experiment was repeated

818

independently 3 times. (B) Growth of WT Lm and $\Delta t c y K$ bacteria in minimal defined

819

media supplemented with different concentrations of reduced glutathione (GSH) as a

820

sole source of cysteine. The data is presented as maximal OD600 values,

821

corresponding to bacterial yield. The data represent 2 biological replicates. Error bars

822

indicate standard deviation.

Fig S8. Intracellular growth of $\Delta t c y K$ and $\Delta g s h F$ mutants in BMDM cells in 825

comparison to WT $\mathbf{L m}$. The experiment was repeated independently 3 times, two 


\section{References}

1. Swaminathan B, Gerner-Smidt P. 2007. The epidemiology of human listeriosis.

Microbes Infect 9:1236-43.

2. Freitag NE, Port GC, Miner MD. 2009. Listeria monocytogenes - from saprophyte to intracellular pathogen. Nat Rev Microbiol 72:623-628.

3. Lecuit M, Cossart P, Pizarro-cerda J. 2003. Invasion of mammalian cells by Listeria monocytogenes : functional mimicry to subvert cellular functions. Trends Cell Biol $13: 23-31$

4. Cossart P, Vicente MF, Mengaud J, Baquero F, Perezdiaz JC, Berche P. 1989.

Listeriolysin-O Is Essential for Virulence of Listeria-Monocytogenes - Direct

5. Smith G a., Marquis H, Jones S, Johnston NC, Portnoy D a., Goldfine H. 1995. The two distinct phospholipases $\mathrm{C}$ of Listeria monocytogenes have overlapping roles in escape from a vacuole and cell-to-cell spread. Infect Immun 63:4231-4237.

6. Marquis H, Doshi V, Portnoy DA. 1995. The broad-range phospholipase C and a metalloprotease mediate listeriolysin O-independent escape of Listeria monocytogenes

7. Grubmüller S, Schauer K, Goebel W, Fuchs TM, Eisenreich W. 2014. Analysis of $4: 156$.

8. Chico-Calero I, Suárez M, González-Zorn B, Scortti M, Slaghuis J, Goebel W,

9. Sauer J-D, Herskovits AA, O'Riordan MXD. 2019. Metabolism of the Gram-Positive Bacterial Pathogen Listeria monocytogenes . Microbiol Spectr 7.

10. Keeney K, Colosi L, Weber W, O’Riordan M. 2009. Generation of Branched-Chain of Listeria monocytogenes. J Bacteriol 191:2187-2196.

11. Tilney LG, Portnoy DA. 1989. Actin Filaments and the Growth, Movement, and Spread of the Intacellular Bacterial Parsite, Listeria monocytogenes. JCell Biol 109:1597-1608.

12. Kocks C, Gouin E, Tabouret M, Berche P, Ohayon H, Cossart P. 1992. L.

13. de las Heras A, Cain RJ, Bielecka MK, Vázquez-Boland J a. 2011. Regulation of

14. Radoshevich L, Cossart P. 2017. Listeria monocytogenes : towards a complete picture of its physiology and pathogenesis. Nat Rev Microbiol 2017161 16:32-46. 
metabolic regulator CodY links Listeria monocytogenes metabolism to virulence by

16. Haber A, Friedman S, Lobel L, Burg-Golani T, Sigal N, Rose J, Livnat-Levanon N, 884 Lewinson O, Herskovits AA. 2017. L-glutamine Induces Expression of Listeria monocytogenes Virulence Genes. PLOS Pathog 13:e1006161.

17. Reniere ML, Whiteley AT, Hamilton KL, John SM, Lauer P, Brennan RG, Portnoy DA. 2015. Glutathione activates virulence gene expression of an intracellular pathogen. Nature 517:170-173.

18. Johansson J, Mandin P, Renzoni a, Chiaruttini C, Springer M, Cossart P. 2002. An 890 RNA thermosensor controls expression of virulence genes in Listeria monocytogenes. $\quad 891$ Cell 110:551-561.

19. Ripio MT, Brehm K, Lara M, Suarez M, Vazquez-Boland JA. 1997. Glucose-1expressed with virulence factors. J Bacteriol1997/11/26. 179:7174-7180.

20. Krypotou E, Scortti M, Grundström C, Oelker M, Luisi BF, Sauer-Eriksson AE, Vázquez-Boland J. 2019. Control of Bacterial Virulence through the Peptide Signature of the Habitat. Cell Rep 26:1815-1827.e5.

21. Lobel L, Sigal N, Borovok I, Ruppin E, Herskovits AA. 2012. Integrative genomic analysis identifies isoleucine and $\operatorname{Cod} \mathrm{Y}$ as regulators of Listeria monocytogenes virulence. PLoS Genet 8:e1002887.

22. Lobel L, Herskovits A a. 2016. Regulatory Activities of CodY Controlling 27.

23. Levdikov VM, Blagova E, Joseph P, Sonenshein AL, Wilkinson AJ. 2006. The structure of CodY, a GTP- and isoleucine-responsive regulator of stationary phase and virulence in gram-positive bacteria. J Biol Chem 281:11366-73.

24. Sonenshein AL. 2005. CodY, a global regulator of stationary phase and virulence in Gram-positive bacteria. Curr Opin Microbiol 8:203-7.

25. Brenner M, Lobel L, Borovok I, Sigal N, Herskovits AA. 2018. Controlled branchedchain amino acids auxotrophy in Listeria monocytogenes allows isoleucine to serve as a host signal and virulence effector. PLoS Genet 14:1-21.

26. Aoyama K, Nakaki T. 2015. Glutathione in Cellular Redox Homeostasis: Association

27. Gopal S, Borovok I, Ofer A, Yanku M, Cohen G, Goebel W, Kreft J, Aharonowitz Y.

28. Hall M, Grundström C, Begum A, Lindberg MJ, Sauer UH, Almqvist F, Johansson J,

29. Wang Y, Feng H, Zhu Y, Gao P. 2017. Structural insights into glutathione-mediated 
30. Tsai HN, Hodgson DA. 2003. Development of a Synthetic Minimal Medium for

31. Xayarath B, Marquis H, Port GC, Freitag NE. 2009. Listeria monocytogenes CtaP is a 927 multifunctional cysteine transport-associated protein required for bacterial 928 pathogenesis. Mol Microbiol 74:956-973.

32. Schauer K, Geginat G, Liang C, Goebel W, Dandekar T, Fuchs TM. 2010.

Deciphering the intracellular metabolism of Listeria monocytogenes by mutant screening and modelling. BMC Genomics 11:573.

33. Friedman S, Linsky M, Lobel L, Rabinovich L, Sigal N, Herskovits AA. 2017.

34. Burguière P, Auger S, Hullo M, Danchin A, Martin-verstraete I, Burguie P. 2004.

Three Different Systems Participate in 1-Cystine Uptake in Bacillus subtilis Three

35. Tanous C, Soutourina O, Raynal B, Hullo MF, Mervelet P, Gilles AM, Noirot P, 941 Danchin A, England P, Martin-Verstraete I. 2008. The CymR regulator in complex 9942 with the enzyme CysK controls cysteine metabolism in Bacillus subtilis. J Biol Chem 943 283:35551-35560.

36. Burguière P, Fert J, Guillouard I, Danchin A, Martin-verstraete I, Burguie P, Auger S.

2005. Regulation of the Bacillus subtilis ytmI Operon, Involved in Sulfur Metabolism.

J Bacteriol 187:6019-6030.

37. CM C, A D, P M, A S. 2014. Paralogous metabolism: S-alkyl-cysteine degradation in Bacillus subtilis. Environ Microbiol 16:101-117.

38. Even S, Burguière P, Auger S, Danchin A, Martin-verstraete I, Burguie P, Soutourina

39. Rees DC, Johnson E, Lewinson O. 2009. ABC transporters: The power to change. Nat

40. Biswas R, Sonenshein AL, Belitsky BR. 2020. Role of GlnR in Controlling

41. Marquis H, Bouwer HGA, Hinrichs DJ, Portnoy DA. 1993. Intracytoplasmic growth 3760 .

42. Reniere ML, Whiteley AT, Portnoy DA. 2016. An In Vivo Selection Identifies Listeria

43. Ji Q, Zhang L, Sun F, Deng X, Liang H, Bae T, He C. 2012. Staphylococcus aureus 964 CymR is a new thiol-based oxidation-sensing regulator of stress resistance and 965 oxidative response. J Biol Chem 287:21102-21109. 
45. Brinsmade SR, Alexander EL, Livny J, Stettner AI, Segrè D, Rhee KY, Sonenshein

46. Combs JA, DeNicola GM. 2019. The Non-Essential Amino Acid Cysteine Becomes

47. Sato H, Tamba M, Ishii T, Bannai S. 1999. Cloning and Expression of a Plasma

48. Kang M, Eichhorn CD, Feigon J. 2014. Structural determinants for ligand capture by a class II preQ1 riboswitch. Proc Natl Acad Sci U S A 111:E663-71.

49. Shih AY, Erb H, Sun X, Toda S, Kalivas PW, Murphy TH. 2006. Cystine/Glutamate

50. Kortebi M, 1๑ M, Mitchell G, Choux CP, Prevost M-C, Cossart P, Bierne H. 2017. 984 Listeria monocytogenes switches from dissemination to persistence by adopting a vacuolar lifestyle in epithelial cells https://doi.org/10.1371/journal.ppat.1006734.

51. Birmingham CL, Canadien V, Kaniuk NA, Steinberg BE, Higgins DE, Brumell JH. 2008. Listeriolysin O allows Listeria monocytogenes replication in macrophage vacuoles. Nat $20084517176451: 350-354$.

52. Wong J, Chen Y, Gan YH. 2015. Host cytosolic glutathione sensing by a membrane

53. Alkhuder K, Meibom KL, Dubail I, Dupuis M, Charbit A. 2009. Glutathione provides a source of cysteine essential for intracellular multiplication of Francisella tularensis. PLoS Pathog 5.

54. Portman JL, Dubinsky SB, Peterson BN, Whiteley AT, Portnoy DA. 2017. Activation

55. Vergauwen B, Elegheert J, Dansercoer A, Devreese B, Savvides SN. 2010.

Glutathione import in Haemophilus influenzae Rd is primed by the periplasmic heme-

56. Vergauwen B, Verstraete K, Senadheera DB, Dansercoer A, Cvitkovitch DG, Guédon

57. Simon R, Priefer U, Pühler A. 1983. A Broad Host Range Mobilization System for In

58. Phan-thanh L, Gormon T. 1997. A chemically defined minimal medium for the 
Reporter System for In Vitro and Organ-Specific Monitoring of Differential Gene

60. Stead MB, Agrawal A, Bowden KE, Nasir R, Mohanty BK, Meagher RB, Kushner SR. 2012. RNAsnap ${ }^{\mathrm{TM}}$ : a rapid, quantitative and inexpensive, method for isolating total RNA from bacteria. Nucleic Acids Res 40:e156.

61. Bailey TL, Boden M, Buske FA, Frith M, Grant CE, Clementi L, Ren J, Li WW,

Noble WS. 2009. MEME Suite: Tools for motif discovery and searching. Nucleic Acids Res 37:202-208.

62. Levdikov VM, Blagova E, Young VL, Belitsky BR, Lebedev A, Sonenshein AL,

63. Solovyev V, Salamov A. 2010. Automatic Annotation of Bacterial Community

64. Wurtzel O, Sesto N, Mellin JR, Karunker I, Edelheit S, Bécavin C, Archambaud C,

65. Crimmins GT, Herskovits AA, Rehder K, Sivick KE, Lauer P, Dubensky TW, Portnoy cytosolic surveillance pathway of innate immunity. Proc Natl Acad Sci U S A

66. Sigal N, Pasechnek A, Herskovits AA. 2016. RNA purification from intracellularly 


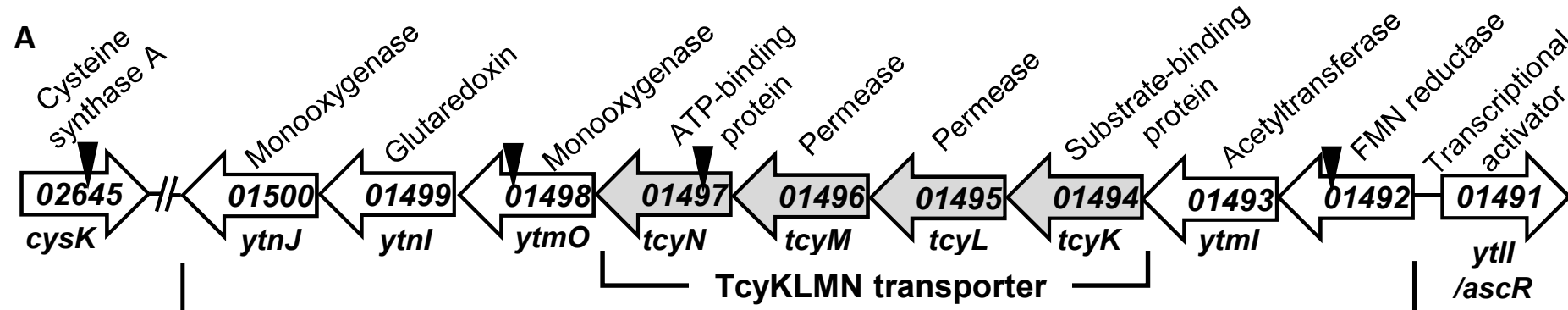

B
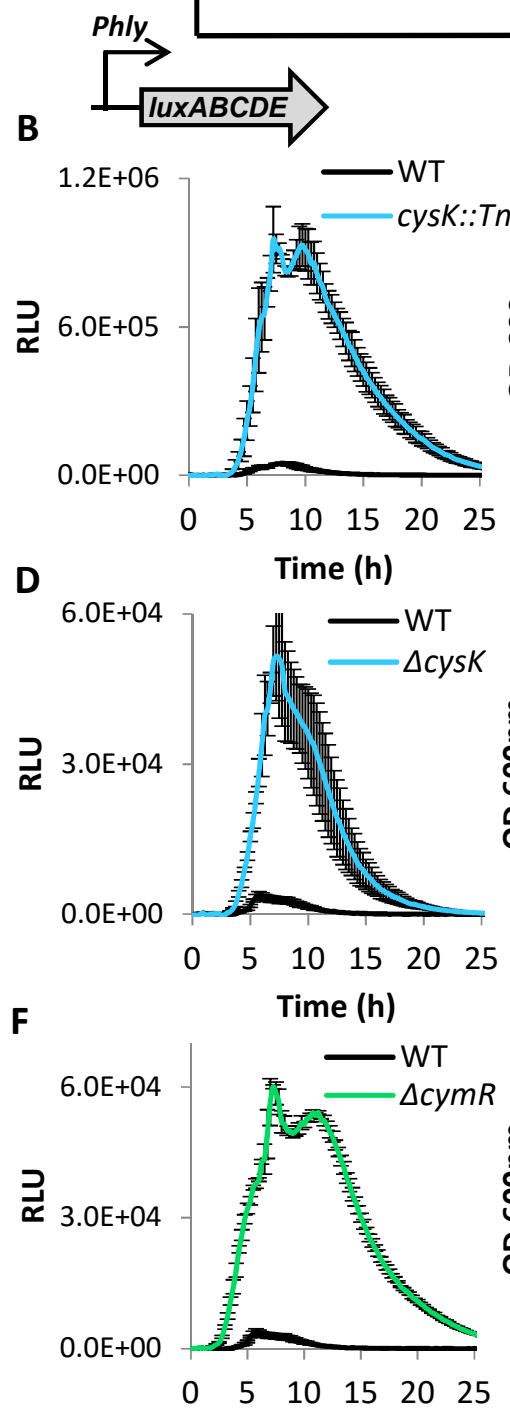

G

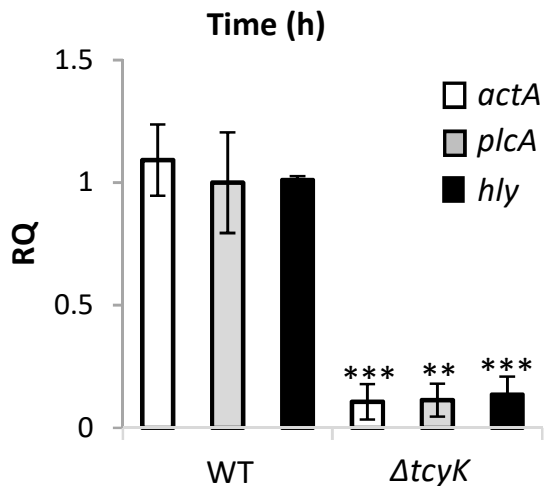

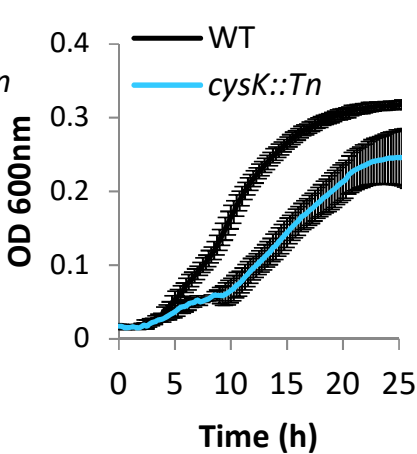
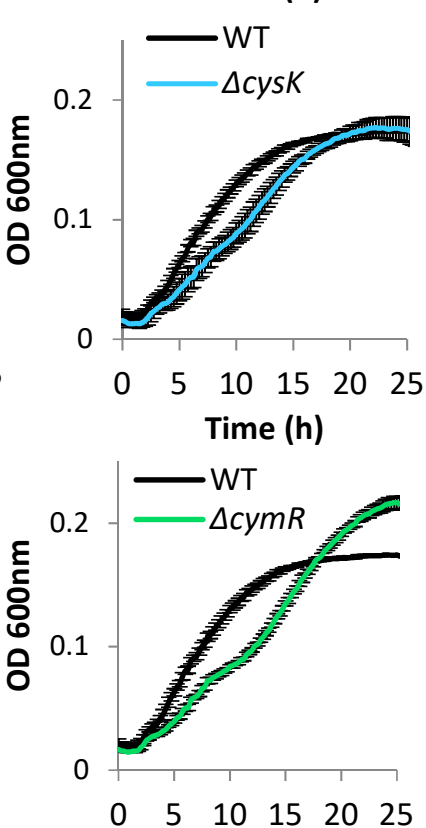

Time (h)

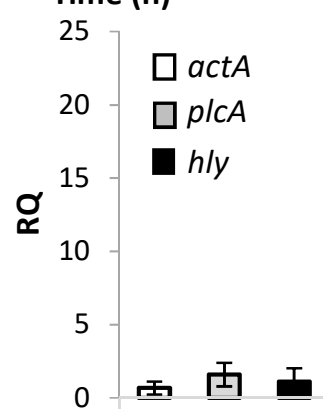

WT

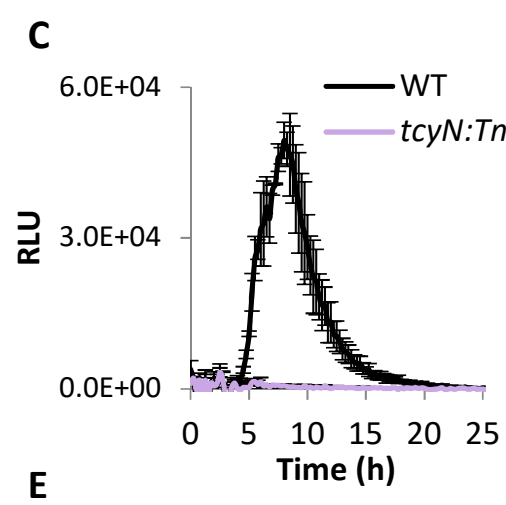

E $6.0 \mathrm{E}+04$
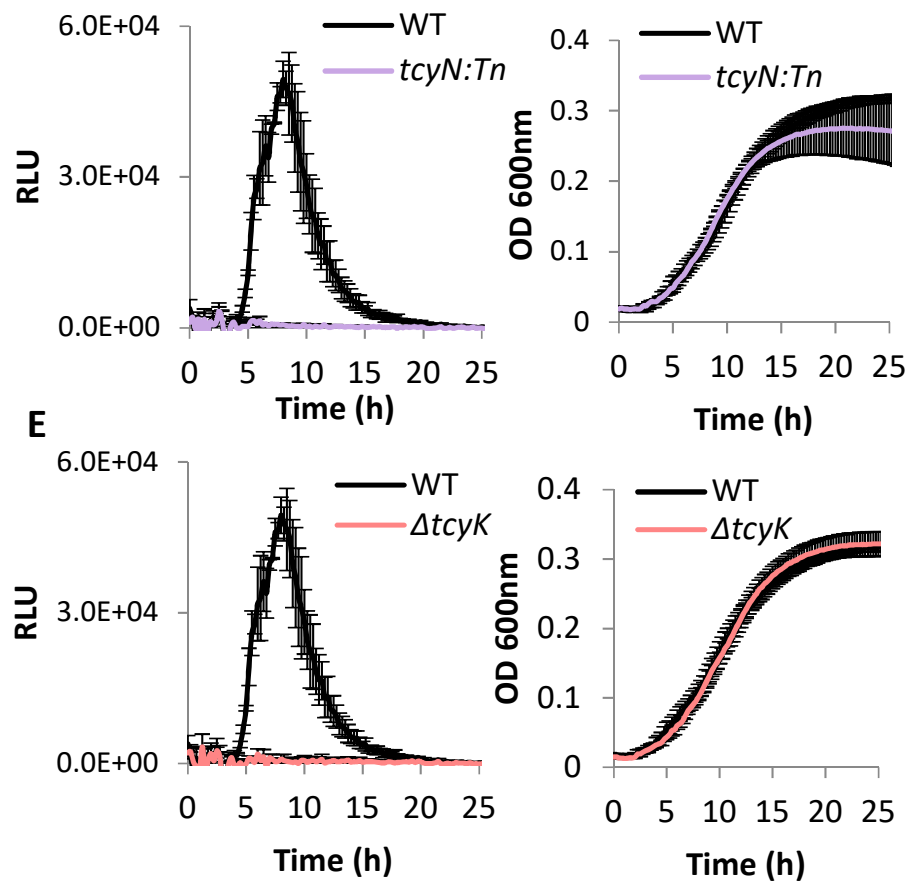

Fig 1. The TcyKLMN transporter and the regulatory factors CysK and CymR play a role in the regulation of $\mathrm{Lm}$ virulence gene expression. See full legend in the next page. 
Fig 1. The TcyKLMN transporter and the regulatory factors CysK and CymR play a role in the regulation of $\mathbf{L m}$ virulence gene expression. (A) A schematic representation of the $y t m /$ operon and the genes identified in Friedman et al. 2017. Locations of transposon insertions are marked with a triangle. (B-F) Luminescence and OD 600nm measurements of bacteria grown in minimal defined media supplemented with low concentrations of branched chain amino acids (LBMM). Relative Luminescence Units (RLU) represent luminescence values normalized to the respective OD $600 \mathrm{~nm}$ value. OD $600 \mathrm{~nm}$ values represent growth. The data represent 3 biological replicates. Error bars indicate standard deviation. (G) qRT-PCR analysis of actA, plcA and hly transcription levels in indicated bacteria grown in LBMM. mRNA levels were normalized to $r p o D$ mRNA and are represented as relative quantity (RQ), relative to mRNA level in WT bacteria. The data represent 3 biological replicates. Error bars indicate standard deviation. Asterisks represent $\mathrm{P}$-values $\left({ }^{*}=\mathrm{P}<0.05,{ }^{* *}=\mathrm{P}<0.01,{ }^{* * *}=\mathrm{P}<0.001\right.$, n.s. $=$ non- significant $)$ calculated by Student's t-test. P-values represent a comparison to the BHI sample. 
A
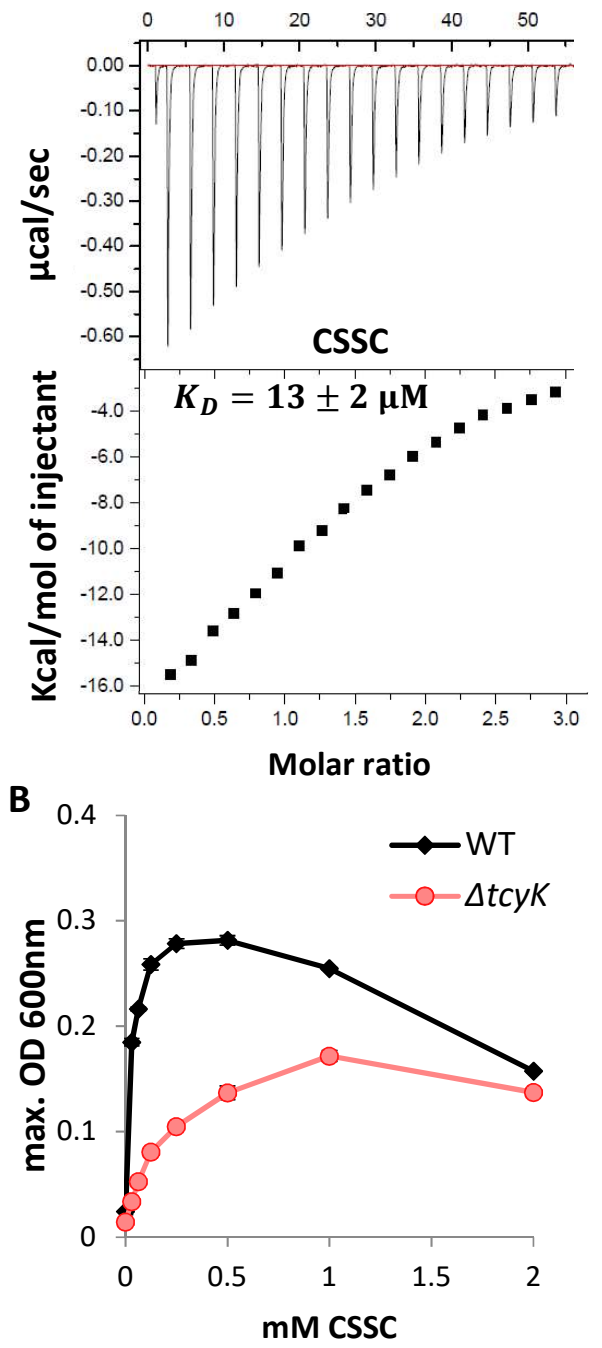

Time (min)
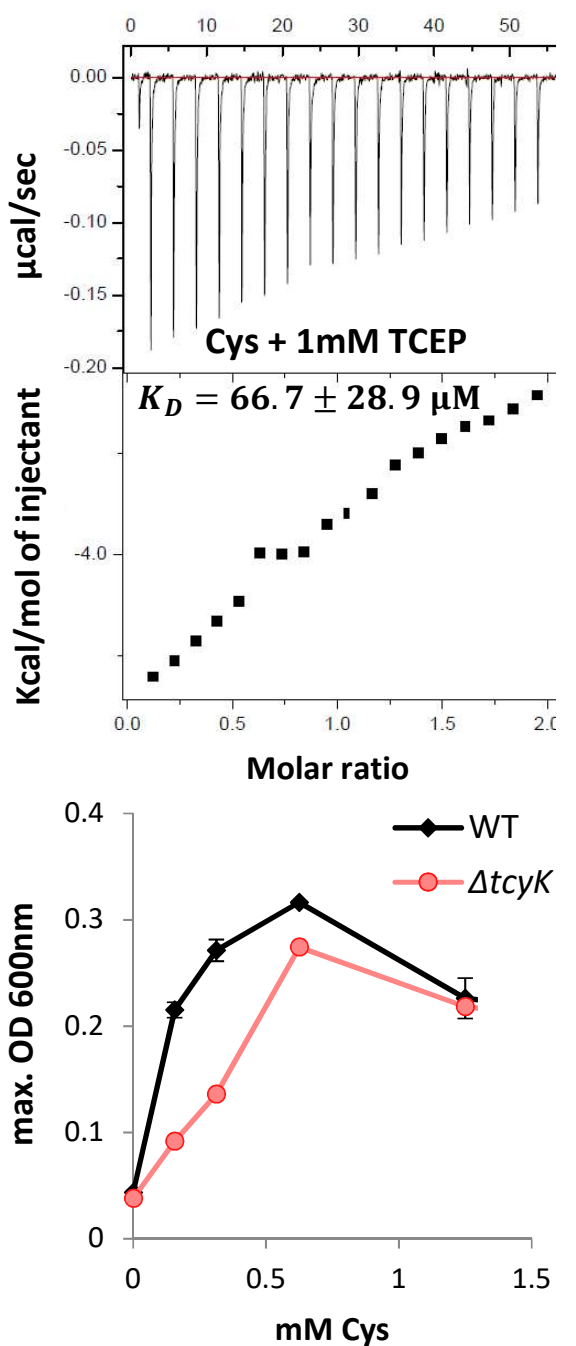

Fig 2. TcyKLMN is a cystine/cysteine transporter. (A) Isothermal Titration Calorimetry (ITC) analysis, showing binding of TcyK to cystine (CSSC) or cysteine (Cys). Shown are the consecutive injections of $2 \mu \mathrm{L}$ aliquots from a $200 \mu \mathrm{M}$ Cys/CSSC solution into $200 \mu \mathrm{L}$ of $20 \mu \mathrm{M}$ TcyK. TCEP was used as a reducing agent when indicated. The upper panels show the calorimetric titration and the lower panels display the integrated injection heat derived from the titrations, for which the best-fit curve was used to calculate the $K_{D}$. The experiments were repeated independently 3 times, and the $K_{D}$ value is presented as a mean \pm standard deviation of these 3 experiments. (B) Growth of WT Lm and $\Delta t c y K$ bacteria grown in minimal defined media supplemented with different concentrations of cysteine (Cys) or cystine (CSSC) as a sole cysteine source. The data is presented as maximal OD $600 \mathrm{~nm}$ values, corresponds to bacterial yield. The data represent 2 biological replicates. The experiment was repeated independently twice, with a total of 4 biological replicates. Error bars indicate standard deviation. 
A

The ytml-ytll regulatory region

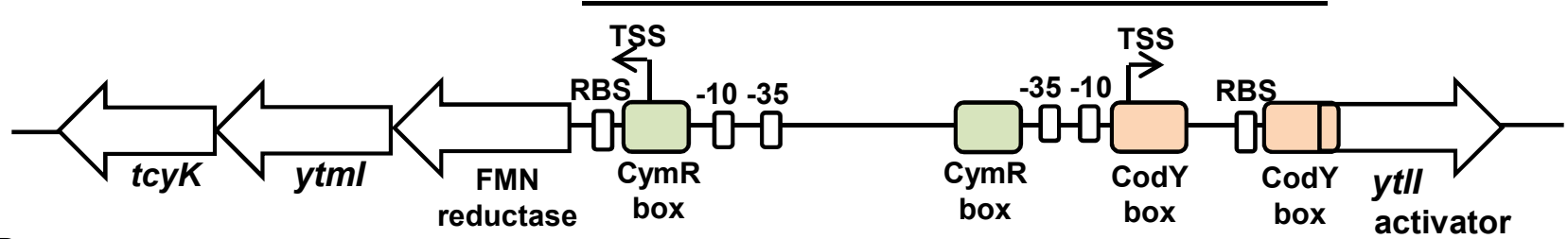

B

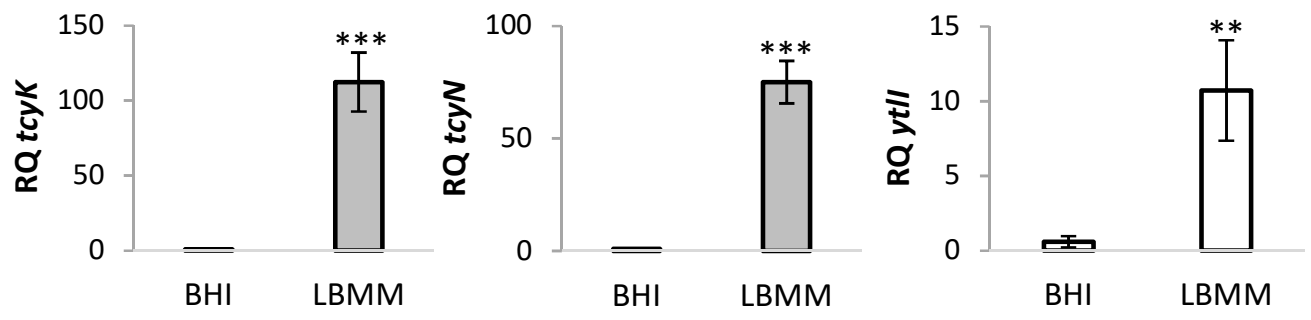

C

BHI

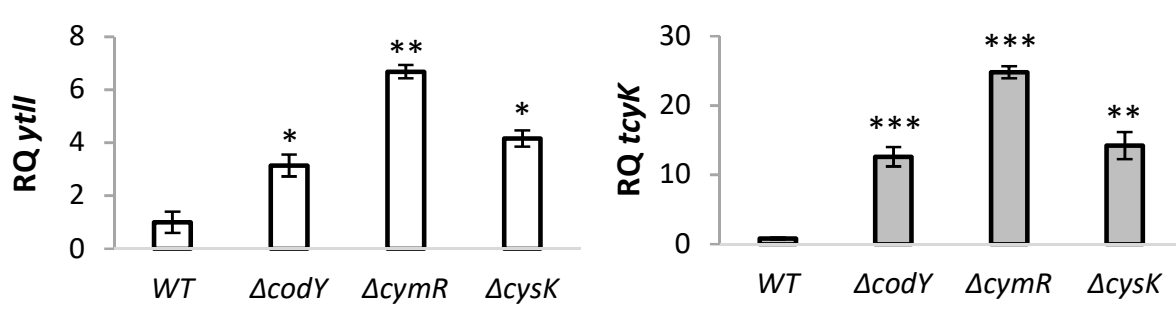

D

\section{LBMM}
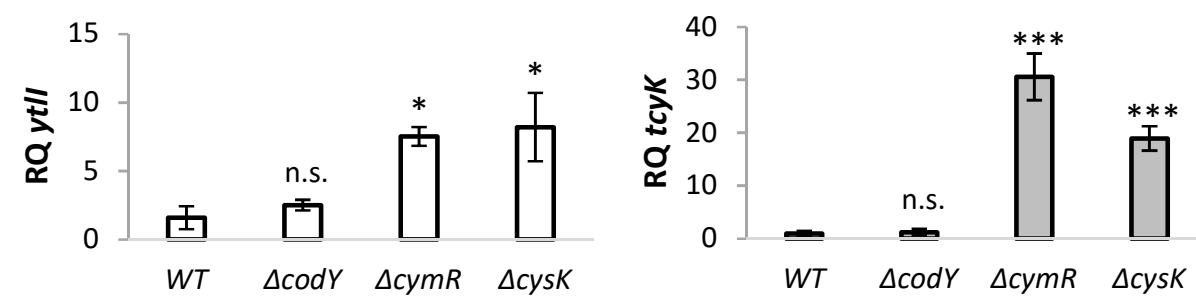

E

\section{$\underline{\text { LCMM }}$}
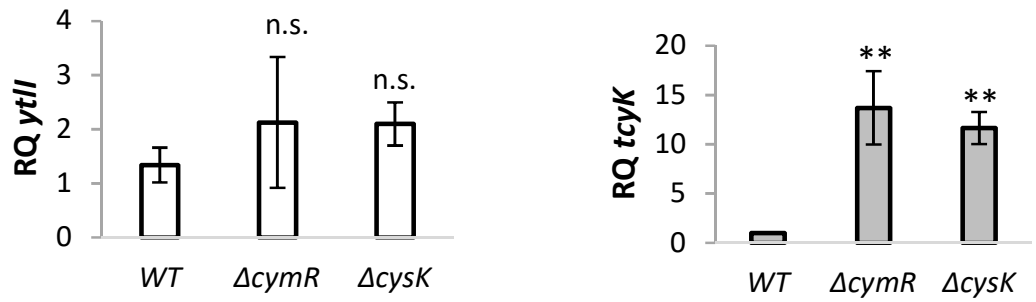

Fig 3. The TcyKLMN transporter is negatively regulated by CodY, CysK and CymR. See full legend in the next page. 
Fig 3. The TcyKLMN transporter is negatively regulated by CodY, CysK and CymR. (A) A schematic representation of the $y t m l-y t / l$ regulatory region. Putative CymR binding-sites were identified using the motif of Bacillus. Putative CodY binding sites were identified using the motif of gram-positive bacteria. Promoters (-10 and -35) were identified using BPROM. TSS denotes the transcription start sites, either identified by Wurtzel et al., 2012, or predicted based on the promoter sequence. (B) qRT$\mathrm{PCR}$ analysis of tcyK, tcyN and $y t / l$ transcription level in WT bacteria grown in rich media (BHI) and in LBMM. mRNA levels were normalized to $r p o D$ mRNA and are represented as relative quantity (RQ), relative to mRNA level in WT bacteria grown in BHI. The data represent 3 biological replicates. Error bars indicate standard deviation. Asterisks represent $\mathrm{P}$-values $\left(^{*}=\mathrm{P}<0.05,{ }^{* *}=\mathrm{P}<0.01, * * *=\mathrm{P}<0.001\right.$, n.s. = non- significant) calculated by Student's t-test. P-values represent a comparison to the BHI sample. (C-E) qRT-PCR analysis of tcyK and $y t / l$ transcription level in the indicated mutants grown in rich media (BHI) and minimal defined media supplemented with low concentration of either branched chain amino acids (LBMM) or cysteine (LCMM). mRNA levels were normalized to rpoD mRNA and are represented as relative quantity (RQ), relative to mRNA level in WT bacteria grown in the indicated medium. The data represent 3 biological replicates. Error bars indicate standard deviation. Asterisks represent $\mathrm{P}$-values $\left(*=\mathrm{P}<0.05,{ }^{* *}=\mathrm{P}<0.01,{ }^{* * *}=\mathrm{P}<0.001\right.$, n.s. $=$ non- significant $)$ calculated by Student's t-test. P-values represent a comparison to the respective WT sample, unless indicated otherwise. 

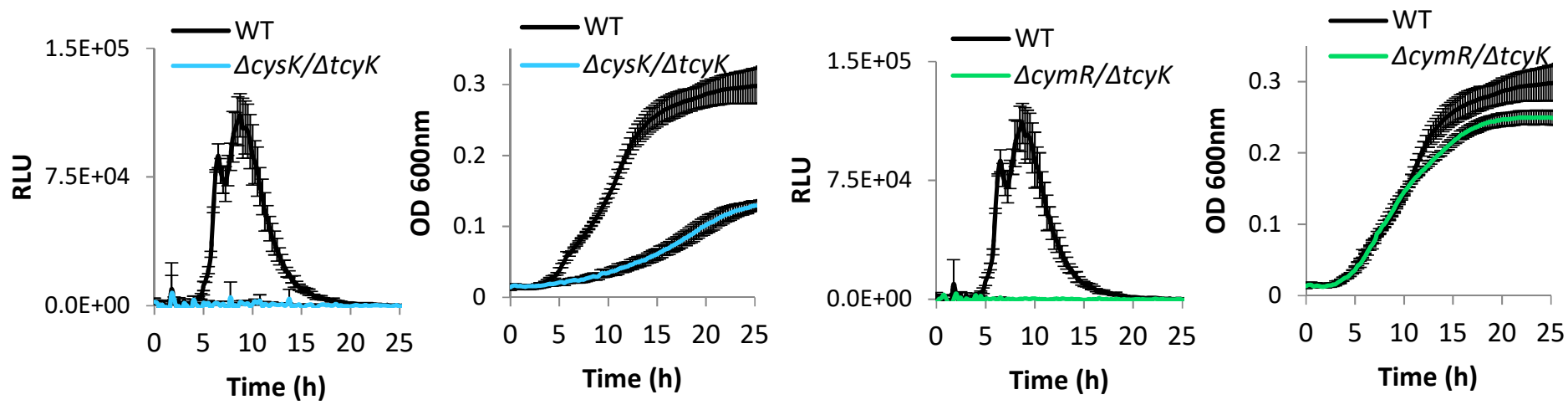

Fig 4. Enhanced expression of virulence genes in $\Delta c y m R$ and $\Delta c y s K$ is TcyKLMN dependent. Luminescence of pPL2 Phly-lux reporter system (left) and growth (right) of $\Delta c y s K / \Delta t c y K$ and $\Delta c y m R / \Delta t c y K$ bacteria grown in minimal defined media supplemented with low concentration of branched chain amino acids (LBMM). Relative Luminescence Units (RLU) represent luminescence values normalized to the respective OD 600nm value. OD 600nm values represent growth. The data represent 3 biological replicates. Error bars indicate standard deviation. 
A

\section{$\underline{\text { LBMM }}$}
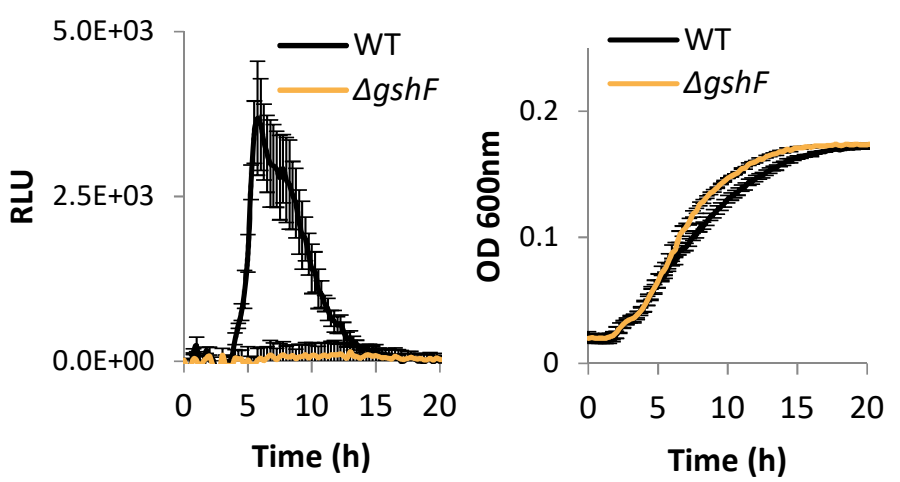

B

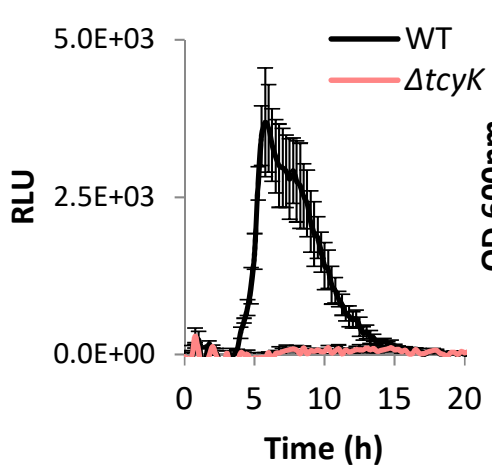

C

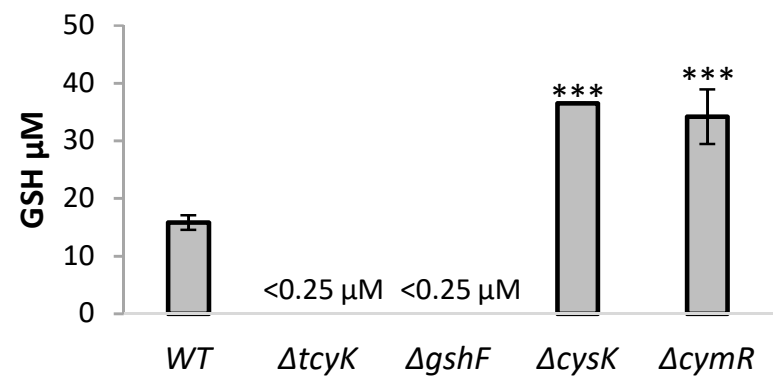

D
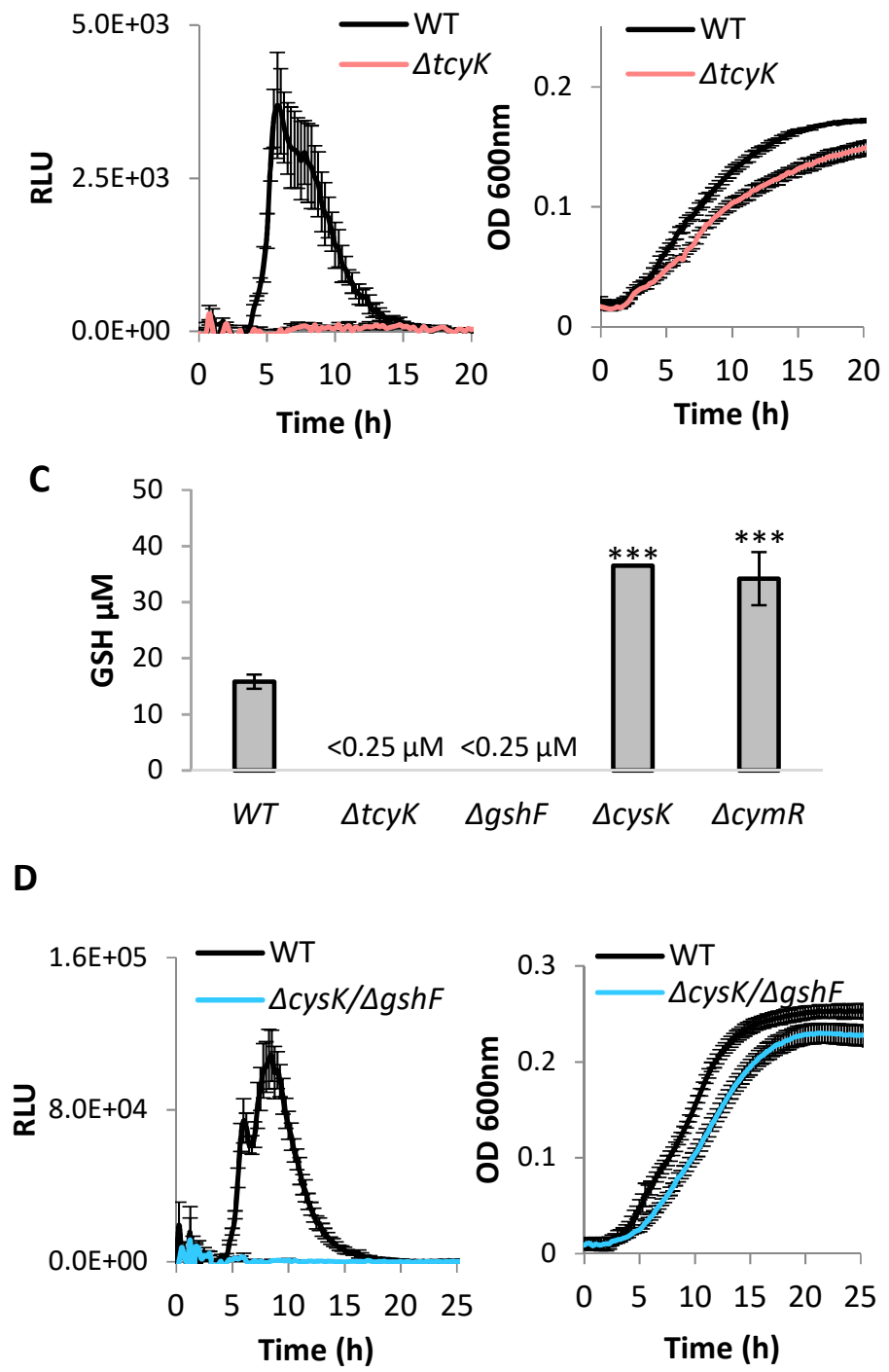

$\underline{\mathrm{LBMM}+20 \mathrm{mM} \mathrm{GSH}}$

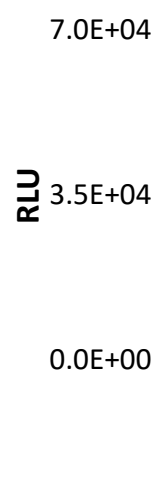

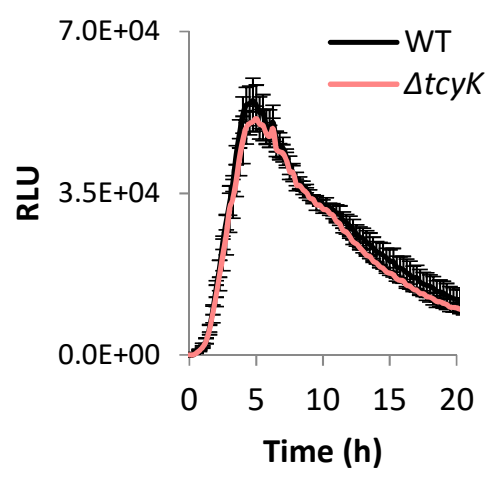
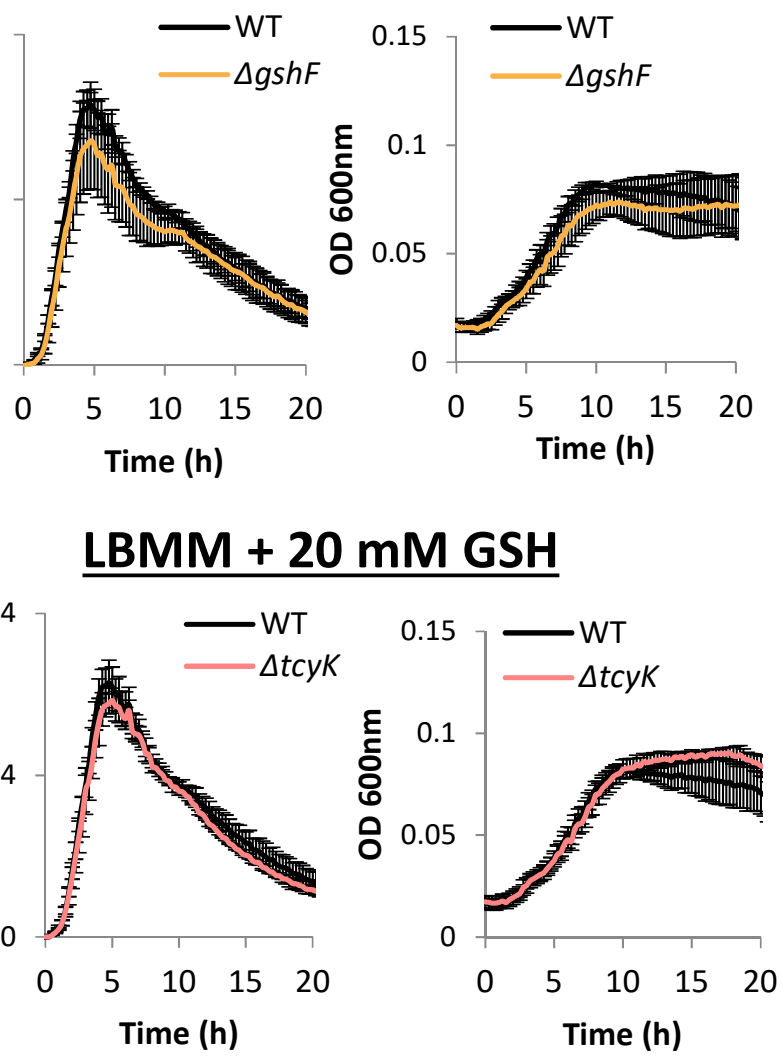

\section{LBMM + $20 \mathrm{mM} \mathrm{GSH}$}
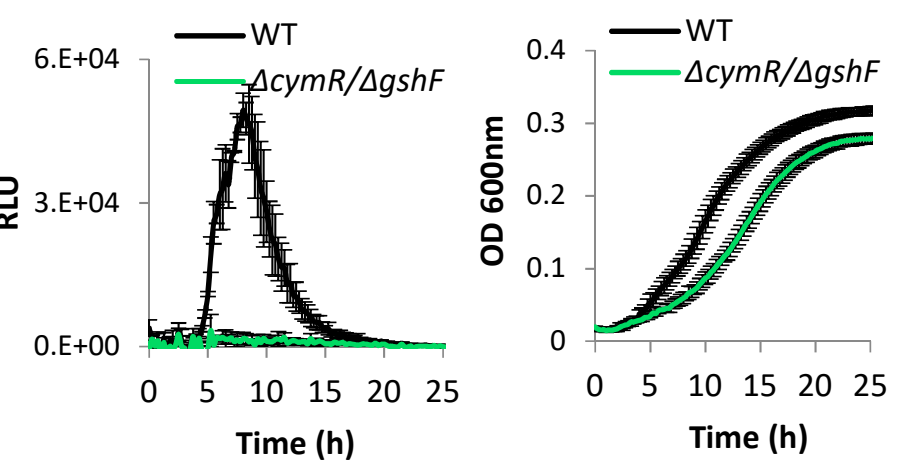

Fig 5. The TcyKLMN transporter plays a role in glutathione synthesis. See full legend in the next page. 
Fig 5. The TcyKLMN transporter plays a role in glutathione biosynthesis. (A-B) Luminescence of pPL2 Phlylux reporter system (left) and growth (right) of $\Delta g s h F$ and $\Delta t c y K$ bacteria grown in minimal defined media with low concentration of branched chain amino acids (LBMM). $20 \mathrm{mM}$ reduced glutathione was supplemented to LBMM when indicated. Relative Luminescence Units (RLU) represent luminescence values normalized to the respective OD $600 \mathrm{~nm}$ value. OD $600 \mathrm{~nm}$ values represent growth. The data represent 3 biological replicates. Error bars indicate standard deviation. (C) Internal glutathione level during growth in LBMM in indicated bacteria. The data represent 3 biological replicates. Error bars indicate standard deviation. Asterisks represent $\mathrm{P}$-values $(* * *=\mathrm{P}<0.001)$ calculated by Student's t-test. P-values represent a comparison to the WT sample. (D) Luminescence of pPL2 Phly-lux reporter system (left) and growth (right) of $\Delta c y s K / \Delta g s h F$ and $\Delta c y m R / \Delta g s h F$ bacteria grown in LBMM. Relative Luminescence Units (RLU) represent luminescence values normalized to the respective OD $600 \mathrm{~nm}$ value. OD $600 \mathrm{~nm}$ values represent growth. The data represent 3 biological replicates. Error bars indicate standard deviation. 
A

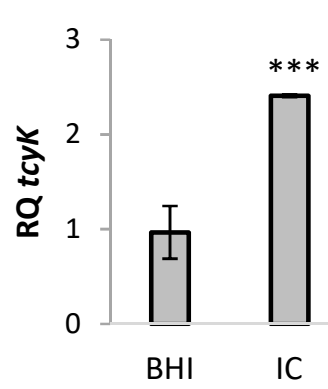

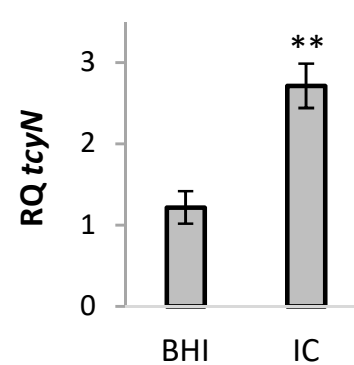
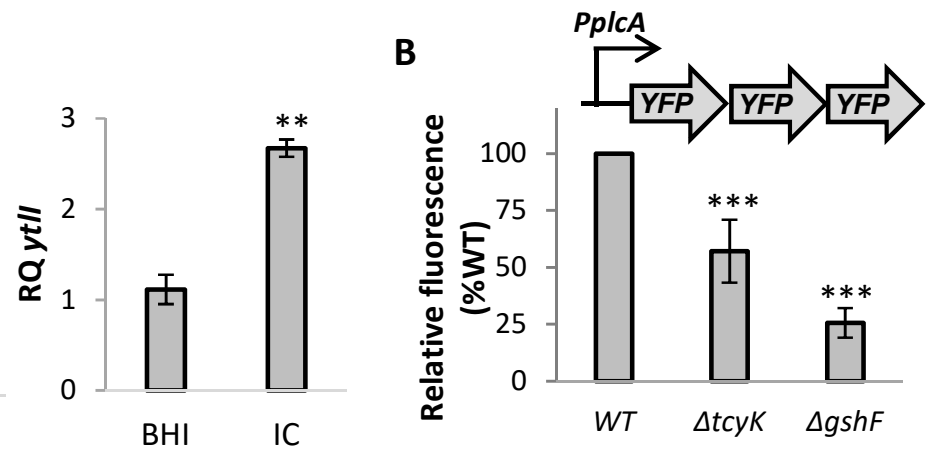

C
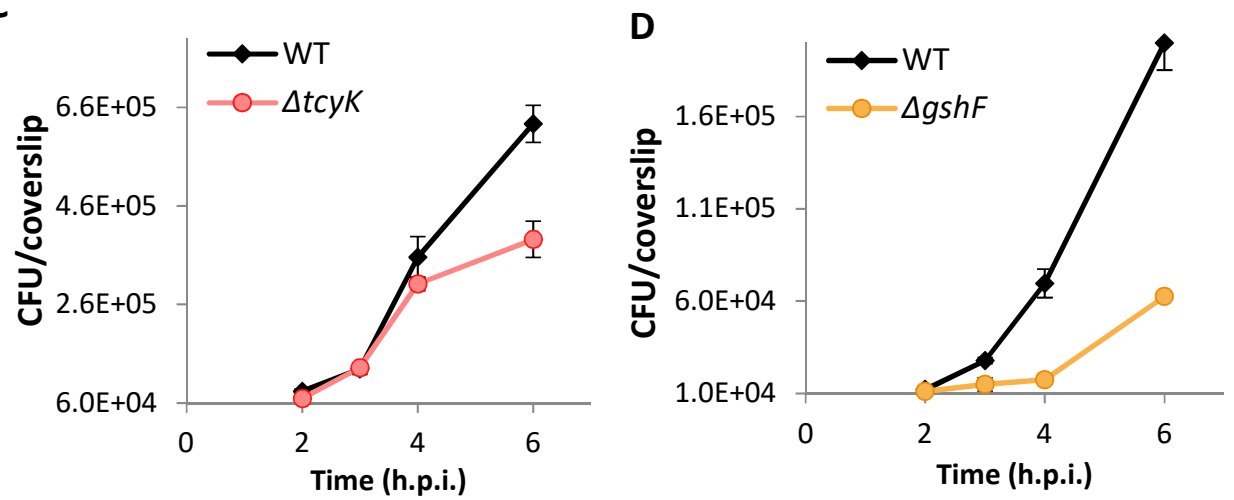

Fig 6. The TcyKLMN transporter promotes $\mathbf{L m}$ intracellular growth in macrophage cells. (A) qRT-PCR analysis of tcyK, tcyN and ytll transcription level in WT bacteria grown in rich media (BHI) or intracellularly in J774A.1 macrophage cell line (IC). mRNA levels were normalized to rpoD mRNA and are represented as relative quantity (RQ), relative to mRNA level in in the BHI sample. The data represent 3 biological replicates. Error bars indicate standard deviation. Asterisks represent P-values $\left(^{*}=\mathrm{P}<0.05,{ }^{* *}=\mathrm{P}<0.01\right.$, ${ }^{* * *}=\mathrm{P}<0.001$, n.s. $=$ non- significant) calculated by Student's t-test. $\mathrm{P}$-values represent a comparison to the BHI sample. (B) Intracellular expression of plcA as indicated by the fluorescence of YFP proteins expressed under the control of $p / c A$ promoter (pPL2-PpICA-3yfp) in WT $L m$ and indicated mutants, grown in bone marrow derived macrophages (BMDM). Fluorescence was measured 3 hours post infection using a florescence microscope. The data represent at least 3 biological replicates. Error bars indicate standard deviation. Asterisks represent $\mathrm{P}$-values $\left({ }^{*}=\mathrm{P}<0.05,{ }^{* *}=\mathrm{P}<0.01, * * *=\mathrm{P}<0.001\right.$, n.s. $=$ non- significant $)$ calculated by Student's t-test. P-values represent a comparison to the WT sample. (C-D) Intracellular growth of $\Delta t c y K$ and $\triangle g s h F$ mutants in BMDM cells in comparison to WT $L m$. The experiment was repeated 3 times independently. Since we could not average the data, a representative result is shown here, and two additional biological repeats are shown in Figure S8. Error bars indicate standard deviation. h.p.i. = hours post infection. 
A

\section{LBMM}
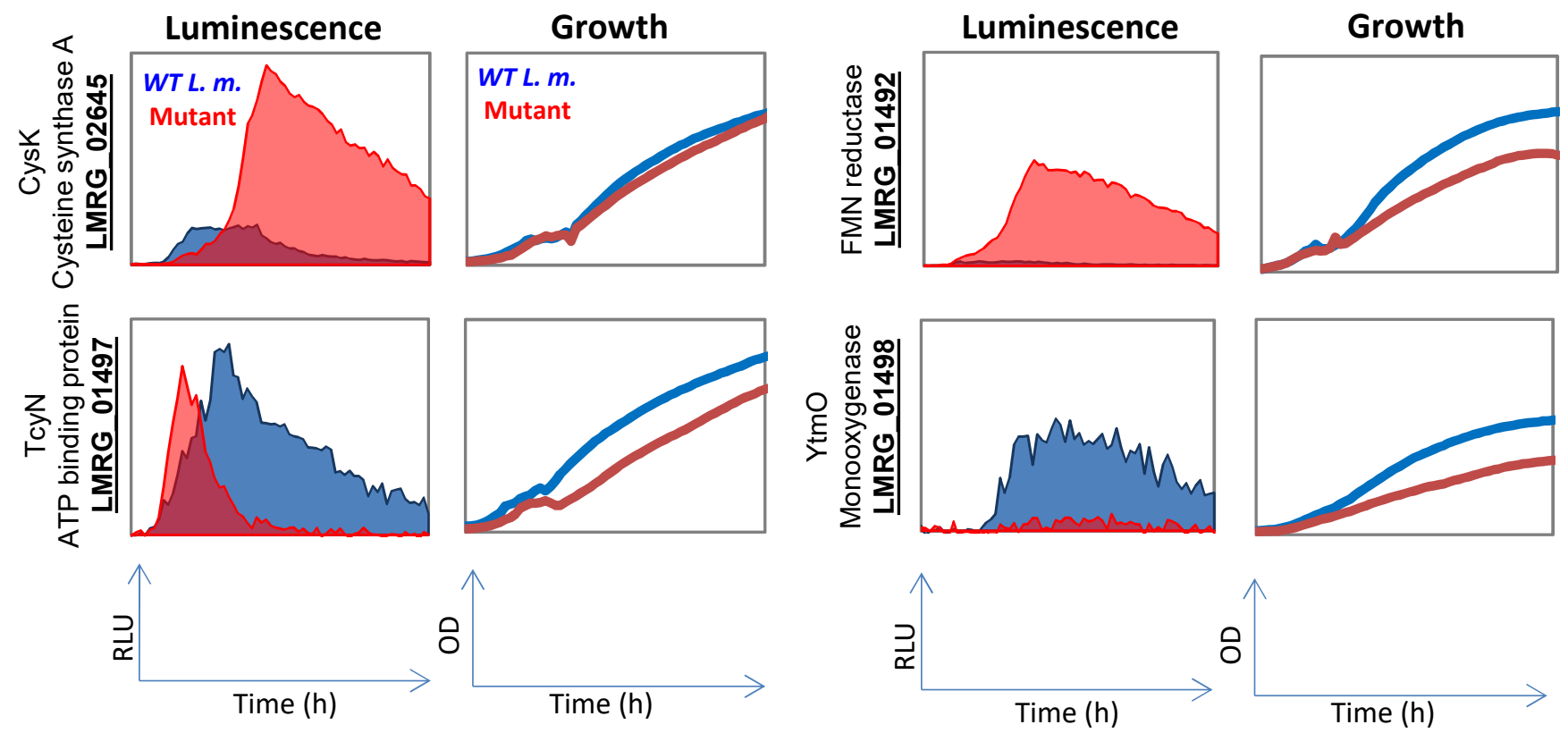

B

\section{Cysteine}

\section{Cystine}

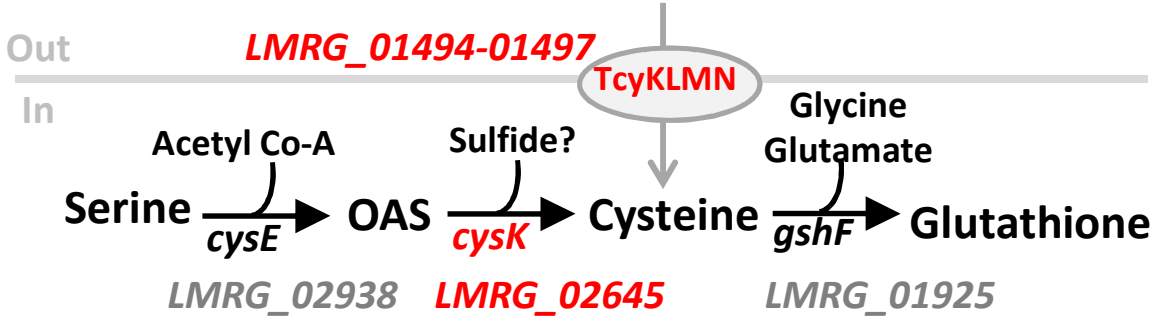

Fig S1. Cysteine uptake and metabolic genes identified in a screen by Friedman et al. (A) Phenotypes of transposon mutants as identified in Friedman et al., 2017. WT bacteria and indicated transposon mutants were grown in minimal defined medium supplemented with low concentration of branched chain amino acids (LBMM). Relative Luminescence Units (RLU) represent luminescence values normalized to the respective OD $600 \mathrm{~nm}$ value. (B) Schematic representation of cysteine transport via TcyKLMN and cysteineglutathione biosynthesis. The genes identified in the genetic screen are marked in red. OAS=Oacetylserine. 
A

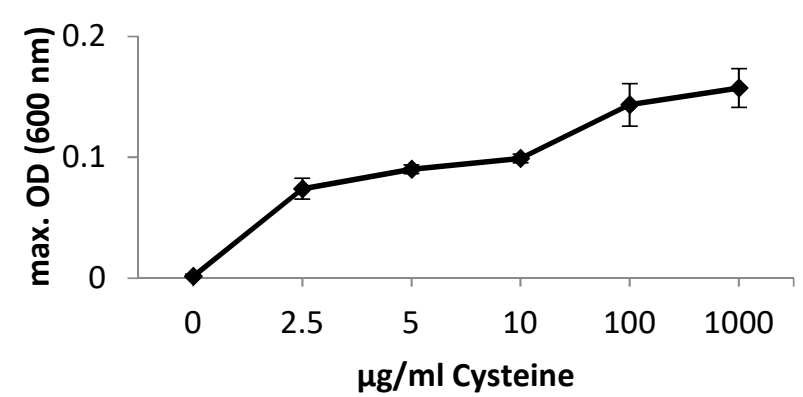

C

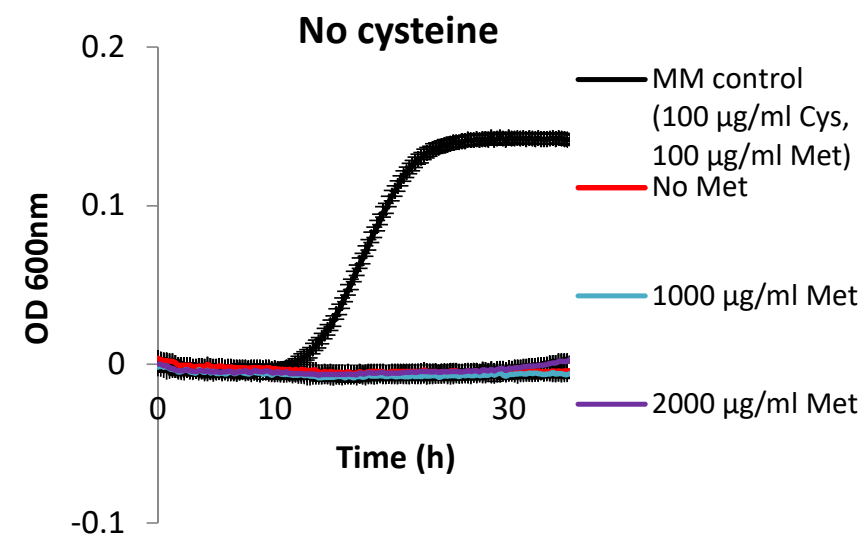

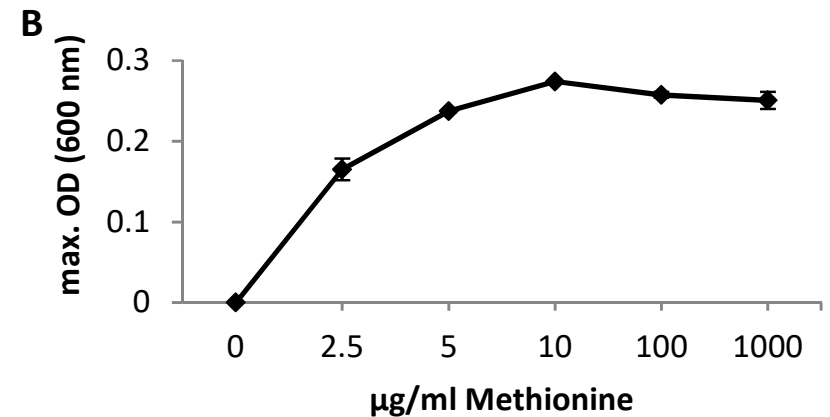

D

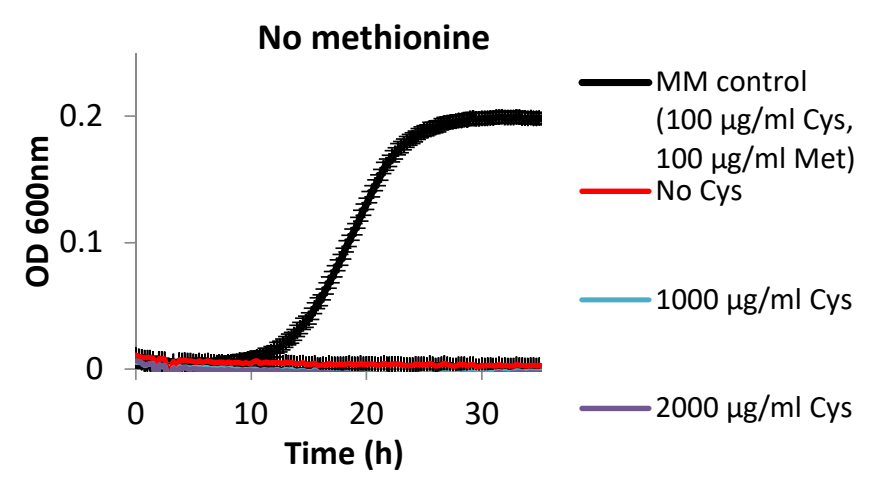

Fig S2. Lm strain 10403S is auxotrophic to cysteine and methionine. (A-B) Growth of WT Lm strain 104035 in minimal defined media supplemented with different concentrations of either cysteine or methionine. The data is presented as maximal OD 600nm values, corresponding to bacterial yield. The data represent 3 biological replicates. Error bars indicate standard deviation. (C) Growth of WT $L m$ in minimal defined media without cysteine supplemented either with $1000 \mu \mathrm{g} / \mathrm{ml}, 2000 \mu \mathrm{g} / \mathrm{ml}$ or no methionine. Growth of WT Lm in minimal defined media supplemented with standard concentrations of cysteine $(100 \mu \mathrm{g} / \mathrm{ml})$ is shown as a control. OD $600 \mathrm{~nm}$ values represent growth. The data represent 3 biological replicates. Error bars indicate standard deviation. (D) Growth of WT $L m$ in minimal defined medium without methionine, supplemented with either $1000 \mu \mathrm{g} / \mathrm{ml}, 2000$ $\mu \mathrm{g} / \mathrm{ml}$ or no cysteine. Growth of WT $L m$ in minimal defined media supplemented with standard concentrations of methionine $(100 \mu \mathrm{g} / \mathrm{ml})$ is shown as a control. OD $600 \mathrm{~nm}$ values represent growth. The data represent 3 biological replicates. Error bars indicate standard deviation. 

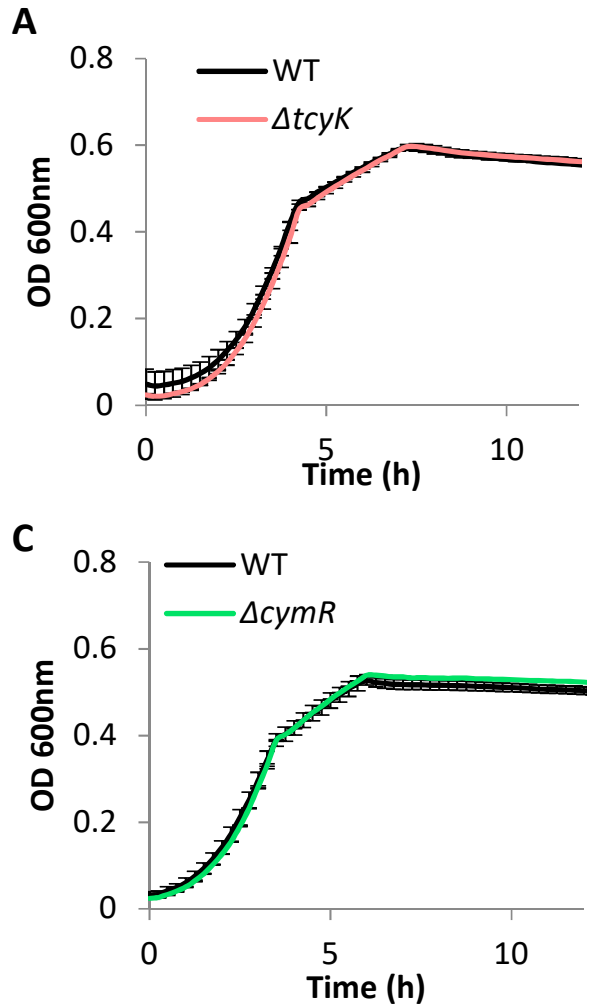

E
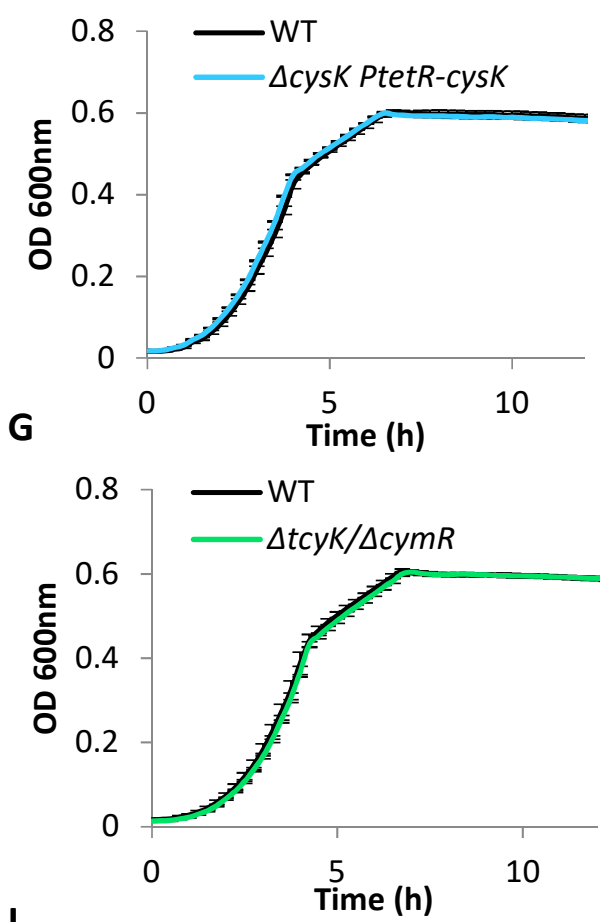

I

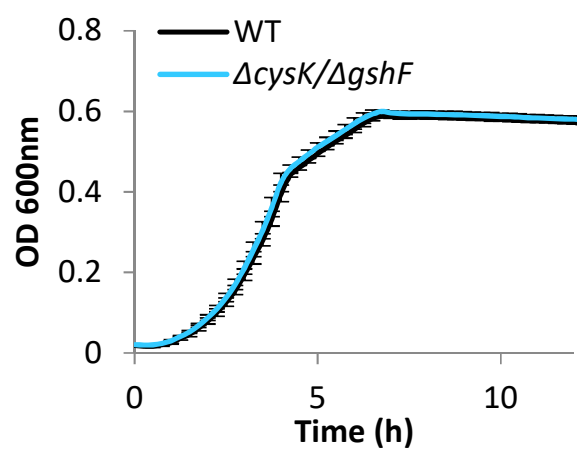

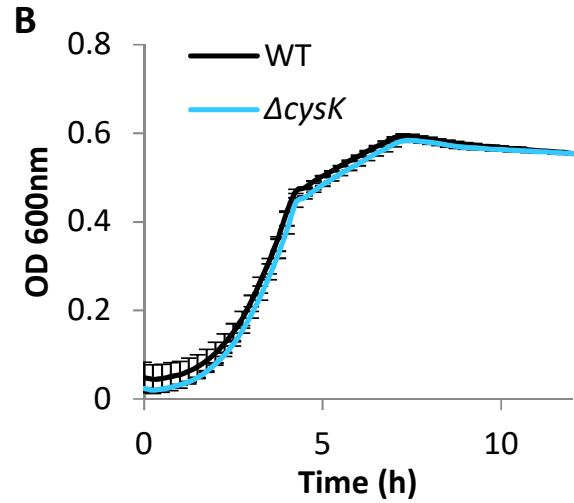

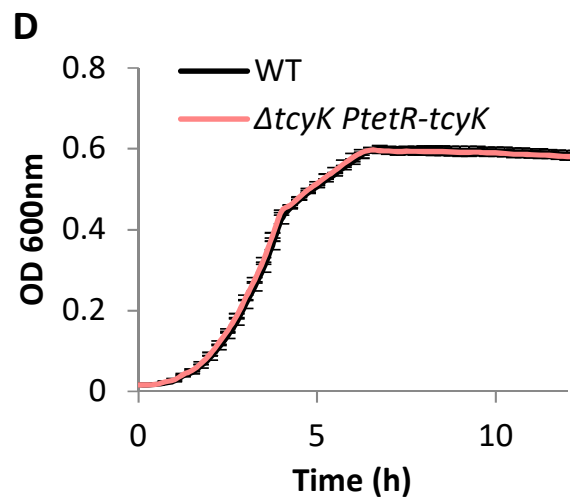

$\mathbf{F}$
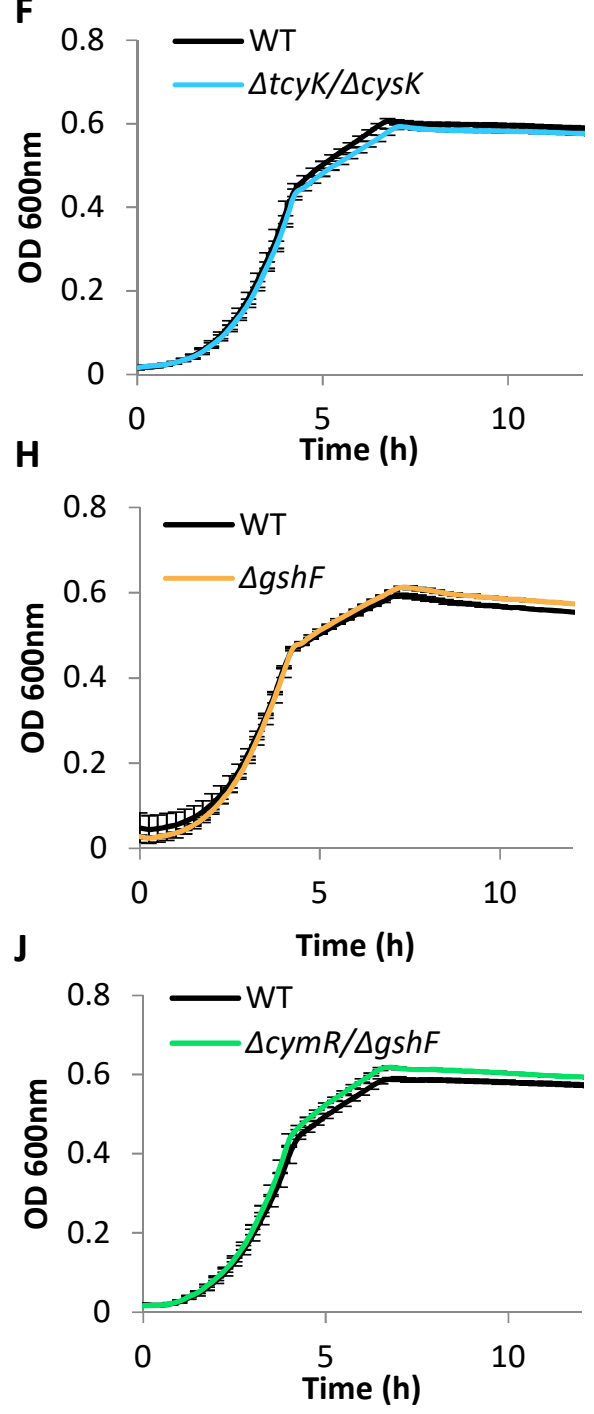

Fig S3. Growth of deletion mutants generated in this study in the rich medium BHI. WT bacteria and indicated strains (A-J) were grown in BHI. OD 600nm values represent growth. The data represent 3 biological replicates. Error bars indicate standard deviation. 


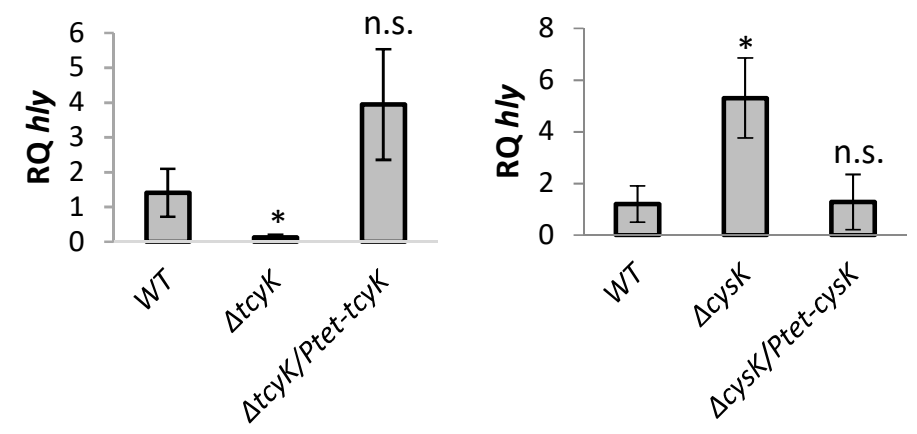

Fig S4. Complementation experiments of $\Delta t c y K$ and $\Delta c y s K$ mutants. qRT-PCR analysis of $h l y$ transcription level in WT $L m, \Delta t c y K$ and $\Delta c y s K$ mutants and their respective complementation strains harboring a copy of tcyK or cys $K$ gene under the tet promoter on pPL2 plasmid, grown in LBMM. mRNA levels were normalized to rpoD mRNA and are represented as relative quantity (RQ), relative to mRNA level in WT bacteria. The data represent at least 2 biological replicates. Error bars indicate standard deviation. Asterisks represent $\mathrm{P}$-values $\left(*=\mathrm{P}<0.05,{ }^{*}=\mathrm{P}<0.01, * * *=\mathrm{P}<0.001\right.$, n.s. $=$ non- significant) calculated by Student's t-test. P-values represent a comparison to the WT sample unless indicated otherwise. 
A

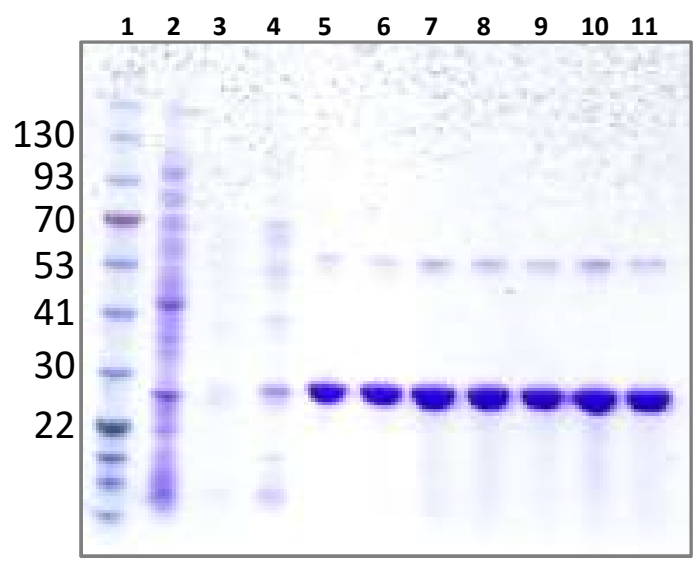

B

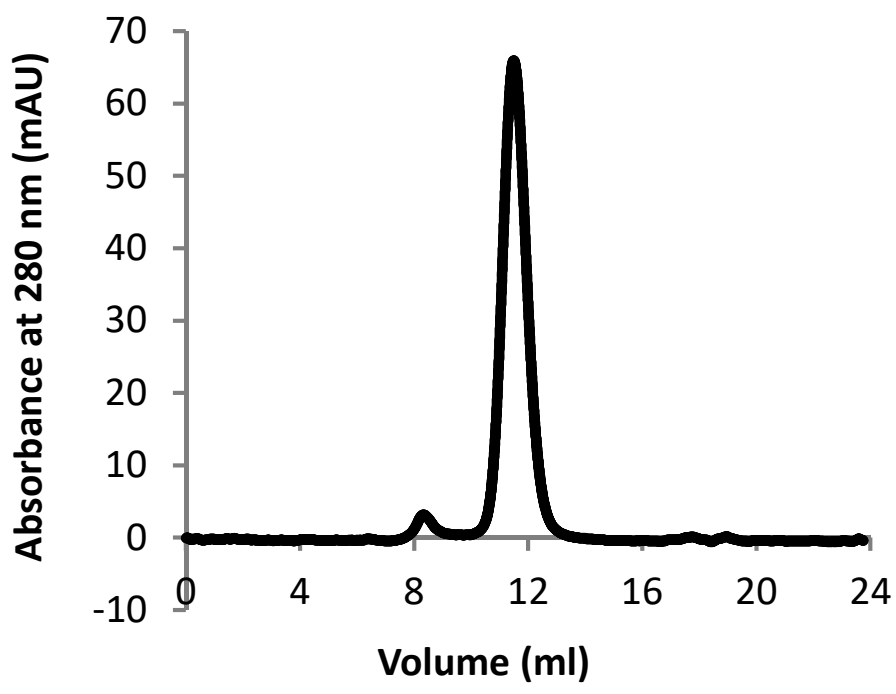

Fig S5. Purification of a short variant of TcyK. (A) Coomassie staining of SDS-PAGE of Ni-NTA affinity purification of a his-tagged short TcyK (amino acids 38-286). Lane 1: molecular weight marker (in kDa), Lane 2: total protein extract, Lane 3: column-unbound fraction, Lane 4: wash with $60 \mathrm{mM}$ of imidazole, Lanes 5-11: fractions eluted using a linear gradient of 60 to $250 \mathrm{mM}$ imidazole. (B) Analysis of the purified protein by size exclusion chromatography. $80 \mu \mathrm{l}$ of a $0.8 \mathrm{mg} / \mathrm{ml}$ protein solution were injected on a Superdex 75 10/300 GL column (GE Healthcare). 
$\underline{\mathrm{BHI}}$

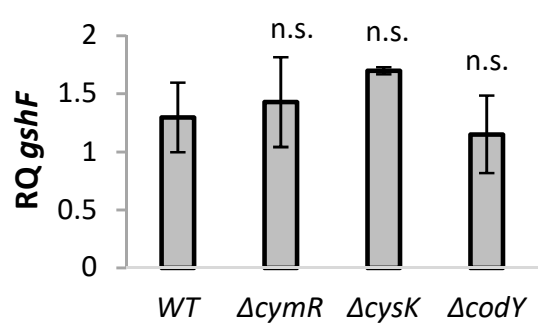

LBMM

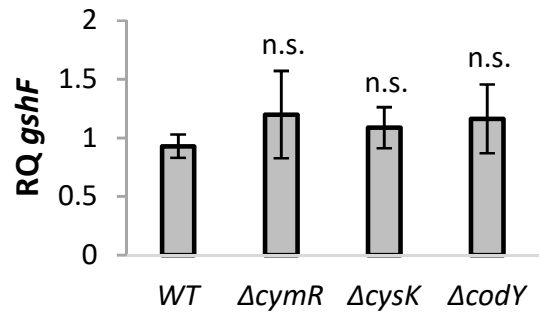

Fig S6. gshF is not transcriptionally regulated by CymR, CysK and CodY. qRT-PCR analysis of gshF transcription level in the indicated mutants grown in rich media (BHI) and minimal defined media supplemented with low concentration of either branched chain amino acids (LBMM). mRNA levels were normalized to $r p o D$ mRNA and are represented as relative quantity (RQ), relative to mRNA level in WT bacteria grown in the indicated medium. The data represent at least 2 biological replicates. Error bars indicate standard deviation Asterisks represent $\mathrm{P}$-values $\left({ }^{*}=\mathrm{P}<0.05,{ }^{* *}=\right.$ $\mathrm{P}<0.01,{ }^{* * *}=\mathrm{P}<0.001$, n.s. $=$ non- significant) calculated by Student's t-test. $\mathrm{P}$-values represent a comparison to the respective WT sample. 
A

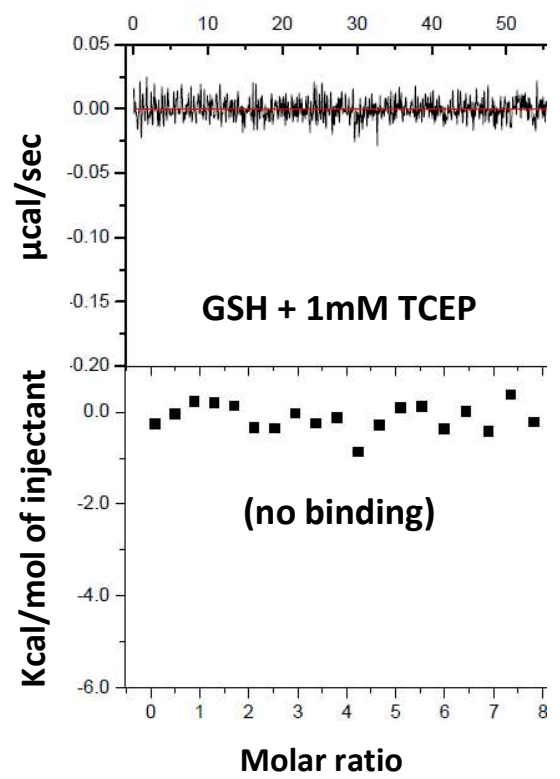

B

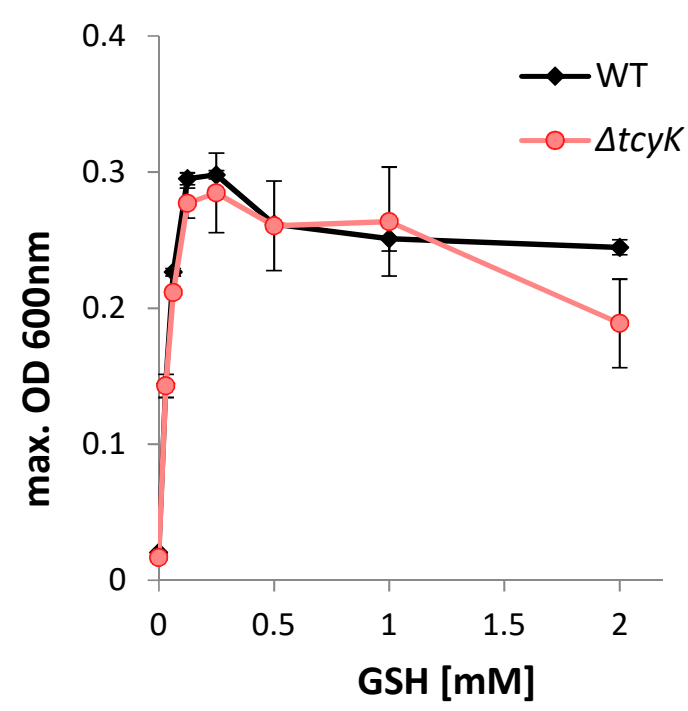

Fig S7. The TCyKLMN transporter does not transport glutathione. (A) Isothermal Titration Calorimetry (ITC) analysis, showing no binding of TcyK to reduced glutathione (GSH). Shown are the consecutive injections of $2 \mu \mathrm{L}$ aliquots from a $200 \mu \mathrm{M}$ GSH solution into $200 \mu \mathrm{L}$ of $20 \mu \mathrm{M}$ TcyK. TCEP was used as a reducing agent. The upper panels show the calorimetric titration and the lower panels display the integrated injection heat derived from the titrations. The experiment was repeated independently 3 times. (B) Growth of WT Lm and $\Delta t c y K$ bacteria in minimal defined media supplemented with different concentrations of reduced glutathione (GSH) as a sole source of cysteine. The data is presented as maximal OD600 values, corresponding to bacterial yield. The data represent 2 biological replicates. Error bars indicate standard deviation. 

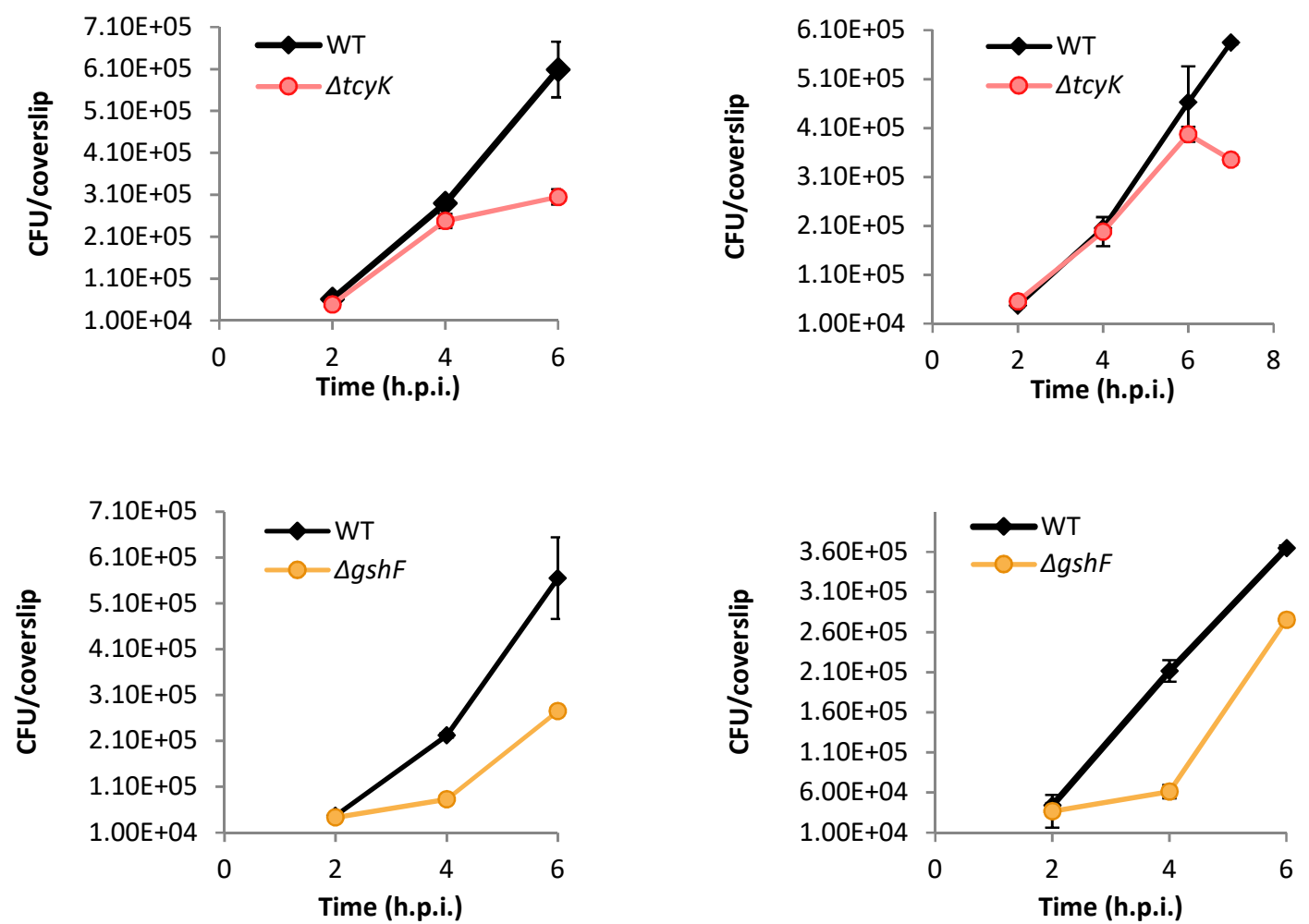

Fig S8. Intracellular growth of $\Delta t c y K$ and $\Delta g s h F$ mutants in BMDM cells in comparison to WT $L m$. The experiment was repeated independently 3 times, two biological repeats for each mutant are shown here, supplementary to Figure 6 C-D. 